J. Bangladesh Acad. Sci., Vol. 42, No. 2, 155-170, 2018

DOI: https://doi.org/10.3329/jbas.v42i2.40042

\title{
SYNTHESIS AND ANTIMICROBIAL ACTIVITY OF 4-OXO-THIAZOLIDINES AND 5- ARYLIDENE DERIVATIVES OF 2-AMINO-5-ETHYL-1,3,4-THIADIAZOLE
}

\author{
SUNIL MAKWANE*, S.D. SRIVASTAVA ${ }^{2}$, RAJIV DUA ${ }^{3}$, AND S.K. SRIVASTAVA ${ }^{4}$ \\ Synthetic Organic Chemistry Laboratory, Department of Chemistry, School of Chemical sciences and \\ Technology, Dr. Harisingh Gour University, Sagar (M.P) India
}

\section{ABSTRACT}

A new series of 5-benzylidene-3-(5-ethyl-[1,3,4]thiadiazol-2-yl)-2-phenyl-thiazolidin-4-ones (3a-3m) were synthesized. The reaction of thioglycolic acid with benzylidene-(5-ethyl-[1,3,4] thiadiazol-2-yl)-amine 1a in the presence of anhydrous $\mathrm{ZnCl}_{2}$ afforded the new heterocyclic compounds 5-benzylidene-3-(5-ethyl-[1,3,4] thiadiazole-yl)-2-phenyl-thiazolidin-4-one, 2a. The latter product on treatment with several selected substituted aromatic aldehydes in the presence of sodium ethoxide underwent the Knoevenagel reaction to yield 5benzylidene-3-(5-ethyl-[1,3,4] thiadiazol-2-yl)-2-phenyl-thiazolidin-4-ones, 3a-3m. The structures of the compounds were confirmed by IR, ${ }^{1} \mathrm{H}-\mathrm{NMR},{ }^{13} \mathrm{C}-\mathrm{NMR}$ and mass spectroscopy and by chemical analysis. All the above compounds were screened for their antimicrobial activity against some selected bacteria and fungi such as E. coli, B. Subtilis, and S. Typhi bacteria and A.niger, A. Flavus and F. oxisporium Fungi.

Key words: Thiadiazole, Benzylidene, Thiazolidinone and Antimicrobial activity

\section{INTRODUCTION}

Heterocycles form by far the largest of classical divisions of organic chemistry and are of immense importance biologically and industrially. The majority of pharmaceuticals and biologically active agrochemicals as well as countless additives and modifiers used in industrial applications ranging from cosmetics, reprography, information storage and plastics are heterocyclic in nature (Dua et al. 2011). The $1,3,4$ - thiadiazole nucleus is one of the most important and well - known heterocyclic nuclei, which is a common and integral feature of a variety of natural products and medicinal agents (Li Y. and Geng J. et al. 2013). Thiadiazole nucleus is present as a core structural component in an array of drug categories such as antimicrobial (Jain A. K. and Sharma S. et al. 2013) anti - inflammatory, analgesic (Shkair A. M. et al. 2016), antinociceptive (Mehlika D. A. et al. 2016), antidepressant, anxiolytic and anticonvulsant agents (Pattanayak P. et al. 2009; Rajak H. et al. 2009), antiviral (Zhuo C. et al. 2010), antineoplastic (Eghbalian A. E. et al. 2013) and antitubercular agents ( Fu X. S. et al. 2016). The broad and potent activity of thiadiazole and their derivatives has established them as pharmacologically significant scaffolds. Thiazolidinones scaffolds are reviewed extensively in literature for its vibrant activities such as antibacterial (Kandapalli V. G. et al. 2010), antifungal (Sharma R. et al. 2012), antioxidant (Parmeshwaran M. et al. 2009), cytotoxic (Jubie S. et al. 2009), antiinflammatory (Goel B. et al. 1999), anticonvulsant (Chaudhary M. et al. 1976), antiHIV (Rawal R. et al. 2005), anti-tubercular (Aamer S. et al. 2007), antiviral activities (Murugesan S. et al. 2012) and DNA Binding

* Corresponding author: <sanumkwn@gmail.com>.

${ }^{2,4}$ Synthetic Organic Chemistry Laboratory, Department of Chemistry, School of Chemical sciences and Technology, Dr. Harisingh Gour University, Sagar (M.P) India.

3 Department of Chemistry, State Forensic Science Laboratory, Department of Home (Police), Goverment of Madhya Pradesh, Sagar (M.P) India. 
Agent (War J. et al. 2017). The incorporation of 4-oxothiazolidine and 5-arylidene moieties in 2amino-5-ethyl-1,3,4-thiadiazole frame work has been found to enhance the activity. Keeping in view the biological importance of the above mentioned heterocyclic compounds and in continuation to our endeavour towards environmentally benign synthesis, we report herein the synthesis of several 3-(5-ethyl$[1,3,4]$ thiadiazole-2-yl)-2-phenyl-thiazolidin-4one (2a-2m) and 3-(5-ethyl-[1,3,4] thiadiazole2-yl)-2-phenyl-thiazolidin-4-one (3a-3m). All the above synthesized compounds were characterized by using spectral techniques such as IR, ${ }^{1} \mathrm{H}$ NMR, ${ }^{13} \mathrm{C}-\mathrm{NMR}$ and $\mathrm{MS}$ spectroscopy. Antibacterial and antifungal activities were performed on E. coli, B. Subtilis, and S. Typhi bacteria and A.niger, A. Flavus and F. oxisporium Fungi.

\section{MATERIALS AND METHODS}

All the chemicals and reagents were of analytical grade of Sigma Aldrich, Merck, Chemi-loba and Himedia. The reagents and solvents were purified before using by standard methods. Melting points were taken in open capillaries and are uncorrected. Progress of reaction was monitored at various stages by silica gel-G coated TLC plates using $\mathrm{MeOH}$ : $\mathrm{CHCl}_{3}$ system. The spot was visualized by exposing dry plate to iodine vapour and fluorescent indicator F 254 UV chamber. IR spectra are recorded in $\mathrm{KBr}$ disc on a Schimadzu 8201 PC, FTIR spectrophotometer ( $v$ max in $\mathrm{cm}^{-1}$ ) and ${ }^{1} \mathrm{H}$ NMR and ${ }^{13} \mathrm{C}$ NMR spectra were measured on a Brucker DRX-300 spectrometer in $\mathrm{CDCl}_{3}$ at 500 and $75 \mathrm{MHz}$ respectively using TMS as an internal standard. All chemical shifts are reported on $\delta$ scale. The mass spectra were recorded on a Jeol SX-102 GC-MS mass spectrometer. Elemental analyses were performed on a Carlo Erba-1108 analyzer. All the synthesized compounds were purified by column chromatography using Merck silica Gel 60 (230-400 Mesh).

Synthesis of Benzylidene- (5-ethyl- [1,3,4] thiadiazol-2-yl)-amine (1a) : The compound 1a was synthesized by standard method using equimolar mixture of 2-amino-5-ethyl-1,3,4thiadiazole $(0.008 \mathrm{~mole})$ and benzaldehyde $(0.008$ mole $)$ in toluene $(25 \mathrm{~mL})$ followed by continuous stirring on a magnetic stirrer for about $1 \mathrm{hr}$. Then the reaction mixture was refluxed on a heating mantle using dean stark apparatus for $2 \mathrm{hrs}$ using glacial acetic acid as a catalyst. Molecular sieves were used to trap excess water molecule in the reaction mixture. After completion of the reaction, the flask was removed from dean stark apparatus and excess solvent was recovered by simple distillation method under reduced pressure at $115-120{ }^{\circ} \mathrm{C}$. A solid product was obtained which was purified over a silica gel column using chloroform: methanol $(8: 2 \mathrm{v} / \mathrm{v})$ mixture as eluant. The elute was concentrated to give a product which was recrystallized from ethanol at room temperature to yield compound 1a: white crystalline solid. m.p. $190-192^{\circ} \mathrm{C}$, yield $75 \%$, IR: 1453( $\left.v_{\mathrm{C}-\mathrm{C}}\right), 712$ $\left(v_{\mathrm{C}-\mathrm{S}}\right), \quad 1670\left(v_{\mathrm{C}=\mathrm{N}}\right), \quad 1642\left(v_{\mathrm{N}=\mathrm{C}-}\right), \quad 1430\left(v_{\mathrm{C}-\mathrm{N}}\right)$, $3118\left(v_{\mathrm{C}-\mathrm{H}}\right), 1310 \quad\left(v_{\mathrm{N}-\mathrm{N}}\right) .{ }^{1} \mathrm{H} \quad \mathrm{NMR}: \quad \delta \quad(\mathrm{ppm})$ $1.20\left(3 \mathrm{H}, \mathrm{CH}_{3} \mathrm{t}, J=7.3 \mathrm{~Hz}\right), 3.06\left(2 \mathrm{H}, \mathrm{CH}_{2}\right.$, $\mathrm{N}=\mathrm{CH} \mathrm{q}, J=7.3 \mathrm{~Hz}), 7.40-8.06(5 \mathrm{H}, \mathrm{Ar}-\mathrm{H}, \mathrm{m}$,$) ,$ $9.39\left(1 \mathrm{H}, \mathrm{s}, \mathrm{CH}\right.$ acyclic; ${ }^{13} \mathrm{C} \mathrm{NMR}: \delta(\mathrm{ppm})$ $12.35\left(\mathrm{CH}_{3}\right.$ acyclic $), 28.93\left(\mathrm{CH}_{2}\right.$ acyclic $)$, 127.7-134.61 (6C of aromatic ring), 158.91 ( $\mathrm{N}=\mathrm{CH}$ acyclic), 159.6 ( $\mathrm{CH}$ acyclic), 156.7, 160.4,156.7 $\left(\mathrm{C}_{2}, \mathrm{C}_{5}\right.$ of thiadiazole $)$; Anal. Calcd for: $\mathrm{C}_{11} \mathrm{H}_{11} \mathrm{~N}_{3} \mathrm{~S}: \mathrm{C}, 60.80, \mathrm{H}, 5.10, \mathrm{~N}, 19.34 \%$; 
found $\mathrm{C}, 60.76, \mathrm{H}, 5.77, \mathrm{~N}, 19.14 \%$; Mass $217\left(\mathrm{M}^{+}\right)$.

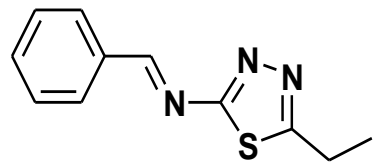

Compoud-1a

The compounds $\mathbf{1 b} \mathbf{- 1} \mathbf{m}$ were synthesized by the similar method as reported for $\mathbf{1 a}$.

\section{2-Chloro-benzylidene-(5-ethyl-[1,3,4]}

thiadiazol-2-yl)-amine (1b): m.p. $175-177{ }^{\circ} \mathrm{C}$, yield 66\%, IR:1453 ( $\left.v_{\mathrm{C}-\mathrm{C}}\right), 742 \quad\left(v_{\mathrm{C}-\mathrm{S}}\right)$, $1647\left(v_{\mathrm{N}=\mathrm{C}}\right), 1430\left(v_{\mathrm{C}-\mathrm{N}}\right), 2989\left(v_{\mathrm{C}-\mathrm{H}}\right), 1310\left(v_{\mathrm{N}-\mathrm{N}}\right)$. ${ }^{1} \mathrm{H}$ NMR: $\delta(\mathrm{ppm}) 1.22\left(3 \mathrm{H}, \mathrm{t}, J=7.3 \mathrm{~Hz}, \mathrm{CH}_{3}\right)$, $3.03(2 \mathrm{H}, \mathrm{q}, J=7.3 \mathrm{~Hz} \mathrm{CH}), 7.35-8.05(4 \mathrm{H}$, m,) $9.41(1 \mathrm{H}, \mathrm{s}, \mathrm{N}=\mathrm{CH}) ;{ }^{13} \mathrm{C} \mathrm{NMR}: \delta(\mathrm{ppm})$ $13.05\left(\mathrm{CH}_{3}\right.$ acyclic $), 27.93 \quad\left(\mathrm{CH}_{2}\right.$ acyclic $)$, 128.8-136.7 (6C of aromatic ring), 159.50 ( $\mathrm{N}=\mathrm{CH}$ acyclic), 156.7, 160.4,156.7 $\left(\mathrm{C}_{2}, \mathrm{C}_{5}\right.$ of thiadiazole); Anal. Calcd for : $\mathrm{C}_{11} \mathrm{H}_{10} \mathrm{~N}_{3} \mathrm{~S}: \mathrm{C}$, 52.48, H, 4.00, N, $16.69 \%$; found C, 52.28, H, 3.97, N, $16.54 \%$; Mass $251\left(\mathrm{M}^{+}\right)$.

\section{(3-Chloro-benzylidene)-(5-ethyl-[1,3,4]}

thiadiazol-2-yl)-amine (1c): m.p.172-175 ${ }^{0} \mathrm{C}$, yield $62 \%$, IR:1543( $\left(v_{\mathrm{C}-\mathrm{C}}\right), 752\left(v_{\mathrm{C}-\mathrm{S}}\right), 1655(\mathrm{v}$ $\mathrm{N}=\mathrm{C}), \quad 1432\left(v_{\mathrm{C}-\mathrm{N}}\right), \quad 3018 \quad\left(v_{\mathrm{C}-\mathrm{H}}\right), 1310 \quad\left(v_{\mathrm{N}-\mathrm{N}}\right)$, $719\left(v_{\mathrm{C}-\mathrm{Cl}}\right) .{ }^{1} \mathrm{H}$ NMR: $\delta(\mathrm{ppm}) 1.20(3 \mathrm{H}, \mathrm{t}, J=7.3$ $\left.\mathrm{Hz} \mathrm{CH}_{3}\right), 3.04\left(2 \mathrm{H}, \mathrm{q}, J=7.3 \mathrm{~Hz} \mathrm{CH}_{2}\right)$, 7.46-7.99 (3H, m), $7.92(1 \mathrm{H}, \mathrm{dt}, \mathrm{J}=7.8 \mathrm{~Hz})$, $9.43\left(1 \mathrm{H}, \mathrm{s}, \mathrm{N}=\mathrm{CH}\right.$ acyclic). ${ }^{13} \mathrm{C} \mathrm{NMR}: \delta(\mathrm{ppm})$ $12.55 \quad\left(\mathrm{CH}_{3}\right.$ acyclic $), 26.93\left(\mathrm{CH}_{2}\right.$ acyclic $)$, 127.8-130.7(C of aromatic ring), 158.91( $\mathrm{N}=\mathrm{CH}$ acyclic),158.4,156.7 $\left(\mathrm{C}_{2}, \mathrm{C}_{5}\right.$ of thiadiazole $)$. Anal. Calcd for : $\mathrm{C}_{11} \mathrm{H}_{10} \mathrm{ClN}_{3} \mathrm{~S}: \mathrm{C}, 52.48, \mathrm{H}$, 4.00, 14.08, N, 16.69; found C,52.18,H,3.92,N,16.50\%; Mass 251( $\left.\mathrm{M}^{+}\right)$.

(4-Chloro-benzylidene)-(5-ethyl-[1,3,4]

thiadiazol-2-yl)-amine (1d): m.p.173-175 ${ }^{0} \mathrm{C}$, yield $67 \%$, IR: 1549( $\left.v_{\mathrm{C}-\mathrm{C}}\right), 746\left(v_{\mathrm{C}-\mathrm{S}}\right), 1661\left(v_{\mathrm{N}}\right.$ $=\mathrm{C}), \quad 1439\left(v_{\mathrm{C}-\mathrm{N}}\right), \quad 3108 \quad\left(v_{\mathrm{C}-\mathrm{H}}\right), 1316\left(v_{\mathrm{N}-\mathrm{N}}\right)$,
$717\left(v_{\mathrm{C}-\mathrm{Cl}}\right) .{ }^{1} \mathrm{H}$ NMR: $\delta(\mathrm{ppm}) 1.26(3 \mathrm{H}, \mathrm{t}, J=7.3$ $\left.\mathrm{Hz} \mathrm{CH}_{3}\right), 3.03\left(2 \mathrm{H}, \mathrm{q}, J=7.3 \mathrm{~Hz} \mathrm{CH}_{2}\right), 7.66-$ $8.00(4 \mathrm{H}, \mathrm{m}, \mathrm{Ar}-\mathrm{H}), 9.37(1 \mathrm{H}, \mathrm{s}, \mathrm{N}=\mathrm{CH}) \cdot{ }^{13} \mathrm{C}$ NMR: $\delta$ (ppm) $12.75\left(\mathrm{CH}_{3}\right.$ acyclic), $27.93\left(\mathrm{CH}_{2}\right.$ acyclic), 129.42-135.7(C of aromatic ring), $158.81(\mathrm{~N}=\mathrm{CH}$ acyclic $), 158.4,156.7\left(\mathrm{C}_{2}, \mathrm{C}_{5}\right.$ of thiadiazole), Anal. Calcd for : $\mathrm{C}_{11} \mathrm{H}_{10} \mathrm{ClN}_{3} \mathrm{~S}: \mathrm{C}$, 52.48, H, 4.00, N, $16.69 \%$; found C, 52.24, H, 3.97, N, $16.51 \%$, Mass; 251( $\left.\mathrm{M}^{+}\right)$.

\section{(2-bromo-benzylidene)-(5-ethyl-[1,3,4]}

thiadiazol-2-yl)-amine (1e): m.p. $180-181^{\circ} \mathrm{C}$, yield $71 \%$, IR:1546(vC-C $), 741\left(v_{\mathrm{C}-\mathrm{S}}\right), 1669\left(\mathrm{v}_{\mathrm{N}}\right.$ $=\mathrm{C}), 1440\left(v_{\mathrm{C}-\mathrm{N}}\right), 2950\left(v_{\mathrm{C}-\mathrm{H}}\right), 1320\left(v_{\mathrm{N}-\mathrm{N}}\right), 549\left(v_{\mathrm{C}-}\right.$ Br). ${ }^{1} \mathrm{H}$ NMR: $\delta(\mathrm{ppm}) 1.20(3 \mathrm{H}, \mathrm{t}, J=6.6 \mathrm{~Hz}$, $\left.\mathrm{CH}_{3}\right), 3.04\left(2 \mathrm{H}, \mathrm{q}, \quad J=6.6 \mathrm{~Hz}, \mathrm{CH}_{2}\right), 7.29-7.91$ (4H, m, ) Ar-H, 9.39 (1H, s, N=CH); ${ }^{13} \mathrm{C}$ NMR: $\delta$ (ppm)12.65( $\mathrm{CH}_{3}$ acyclic), $28.83\left(\mathrm{CH}_{2}\right.$ acyclic $)$, $158.51 \quad(\mathrm{~N}=\mathrm{CH}$ acyclic), 127.42-132.19(Cof aromatic ring), 159.4,155.7 $\left(\mathrm{C}_{2}, \mathrm{C}_{5}\right.$ of thiadiazole $)$, Anal. Calcd for: $\mathrm{C}_{11} \mathrm{H}_{10} \mathrm{Br} \mathrm{N} \mathrm{N}_{3} \mathrm{~S}: \mathrm{C}, 44.61, \mathrm{H}$, $3.40, \mathrm{~N}, 14.19 \%$; found $\mathrm{C}, 44.21, \mathrm{H}, 3.27, \mathrm{~N}$, $14.11 \%$; Mass $294\left(\mathrm{M}^{+}\right)$.

\section{(3-bromo-benzylidene)-(5-ethyl-[1,3,4]}

thiadiazol-2-yl)-amine (1f): m.p.181-182 ${ }^{\circ} \mathrm{C}$, yield $72 \%$, IR:1548( $\left.v_{\mathrm{C}-\mathrm{C}}\right), 750\left(v_{\mathrm{C}-\mathrm{S}}\right), 1645(\mathrm{v}$ $\mathrm{N}=\mathrm{C}), \quad 1438\left(v_{\mathrm{C}-\mathrm{N}}\right), \quad 2952 \quad\left(v_{\mathrm{C}-\mathrm{H}}\right), 1320\left(v_{\mathrm{N}-\mathrm{N}}\right)$, $551\left(v_{\mathrm{C}-\mathrm{Br}}\right) ;{ }^{1} \mathrm{H}$ NMR: $\delta$ (ppm) $1.21 \quad(3 \mathrm{H}, \mathrm{t}$, $\left.J=7.3 \mathrm{~Hz}, \mathrm{CH}_{3}\right), 3.08\left(2 \mathrm{H}, \mathrm{q}, J=7.3 \mathrm{~Hz}, \mathrm{CH}_{2}\right)$, 7.45 -7.96 (4H, m, Ar-H), 9.42 (1H, s, N=CH); ${ }^{13} \mathrm{C}$ NMR $\delta$ (ppm) $12.75\left(\mathrm{CH}_{3}\right.$ acyclic), 27.93 $\left(\mathrm{CH}_{2}\right.$ acyclic), 128.41-133.19(C of aromatic ring), $159.41 \mathrm{~N}=\mathrm{CH}$ acyclic, $156.4,158.7\left(\mathrm{C}_{2}, \mathrm{C}_{5}\right.$ of thiadiazole), Anal. Calcd for: $\mathrm{C}_{11} \mathrm{H}_{10} \mathrm{Br} \mathrm{N}_{3} \mathrm{~S}$ : C, 44.61, H, 3.40, N, $14.19 \%$; found C, 44.21, $\mathrm{H}, 3.27, \mathrm{~N}, 14.15 \%$; Mass $294\left(\mathrm{M}^{+}\right)$.

\section{(4-bromo-benzylidene)-(5-ethyl-[1,3,4]}

thiadiazol-2-yl)-amine (1g): m.p.182-183 ${ }^{\circ} \mathrm{C}$, yield $68 \%$, IR : 1550( $\left(v_{\mathrm{C}-\mathrm{C}}\right), 752\left(v_{\mathrm{C}-\mathrm{S}}\right)$, $1667\left(v_{\mathrm{N}=\mathrm{C}}\right), 1441\left(v_{\mathrm{C}-\mathrm{N}}\right), 3072\left(v_{\mathrm{C}-\mathrm{H}}\right), 1316\left(v_{\mathrm{N}-\mathrm{N}}\right)$, 549 $\left(v_{\mathrm{C}-\mathrm{Br}}\right)$; ${ }^{1} \mathrm{H}$ NMR: $\delta(\mathrm{ppm}) 1.26(3 \mathrm{H}, \mathrm{t}, J=7.3$ $\left.\mathrm{Hz}, \mathrm{CH}_{3}\right), 3.06\left(2 \mathrm{H}, \mathrm{q}, J=7.3 \mathrm{~Hz} \mathrm{CH}_{2}\right), 7.67-$ 
$7.99(4 \mathrm{H}, \mathrm{m}, \mathrm{Ar}-\mathrm{H}), 9.42(1 \mathrm{H}, \mathrm{s}, \mathrm{N}=\mathrm{CH}) .{ }^{13} \mathrm{C}$ NMR: $\delta$ (ppm) $13.76\left(\mathrm{CH}_{3}\right.$ acyclic $), 29.73\left(\mathrm{CH}_{2}\right.$ acyclic), 124.09-134.59 (C of aromatic ring), $158.92(\mathrm{~N}=\mathrm{CH}$ acyclic $), 158.4,159.9\left(\mathrm{C}_{2}, \mathrm{C}_{5}\right.$ of thiadiazole), Anal. Calcd for: $\mathrm{C}_{11} \mathrm{H}_{10} \mathrm{Br} \mathrm{N}_{3} \mathrm{~S}$ : C, 44.61, H, 3.40, N, $14.19 \%$; found C, 44.21, H, 3.19, N, $14.11 \%$; Mass 294( $\left(\mathrm{M}^{+}\right)$.

(2-nitro-benzylidene)-(5-ethyl-[1,3,4]thiadiazol2-yl)-amine (lh): m.p. $176-179^{\circ} \mathrm{C}$, yield $80 \%$, IR:1552( $\left.v_{\mathrm{C}-\mathrm{C}}\right), 753\left(v_{\mathrm{C}-\mathrm{S}}\right), 1649\left(v_{\mathrm{N}=\mathrm{C}}\right), 1436\left(v_{\mathrm{C}-}\right.$ $\mathrm{N}), 3090\left(v_{\mathrm{C}-\mathrm{H}}\right), 1321\left(v_{\mathrm{N}-\mathrm{N}}\right), 1518\left(v \mathrm{C}-\mathrm{NO}_{2}\right)^{1} \mathrm{H}$ NMR: $\delta 1.21\left(3 \mathrm{H}, \mathrm{t}, J=7.1 \mathrm{~Hz}, \mathrm{CH}_{3}\right), 3.06(2 \mathrm{H}$, $\left.\mathrm{q}, J=7.1 \mathrm{~Hz}, \mathrm{CH}_{2}\right), 7.29-7.89(4 \mathrm{H}, \mathrm{m}, \mathrm{Ar}-\mathrm{H}$, $9.39(1 \mathrm{H}, \quad \mathrm{s}, \quad \mathrm{N}=\mathrm{CH}) ;{ }^{13} \mathrm{C}$ NMR: $\delta(\mathrm{ppm})$ 12.55( $\mathrm{CH}_{3}$ acyclic), 26.53( $\mathrm{CH}_{2}$ acyclic), 127.4.148.40 (C of aromatic ring), $160.10(\mathrm{~N}=\mathrm{CH}$ acyclic), 157.4,159.8 $\left(\mathrm{C}_{2}, \mathrm{C}_{5}\right.$ of thiadiazole ring). Anal. Calcd for: $\mathrm{C}_{11} \mathrm{H}_{10} \mathrm{~N}_{4} \mathrm{O}_{2} \mathrm{~S}: \mathrm{C}, 50.37$, $\mathrm{H}, 3.84 \mathrm{~N}, 21.36 \%$; found $\mathrm{C}, 50.22, \mathrm{H}, 3.59, \mathrm{~N}$, $21.16 \%$; Mass $262\left(\mathrm{M}^{+}\right)$.

(3-nitro-benzylidene)-(5-ethyl-[1,3,4]thiadiazol2-yl)-amine (1i) :m.p. $175-177^{\circ} \mathrm{C}$, yield $79 \%$, IR:1549( $\left.v_{\mathrm{C}-\mathrm{C}}\right), 755\left(v_{\mathrm{C}-\mathrm{S}}\right), 1647\left(v_{\mathrm{N}=\mathrm{C}}\right), 1436\left(v_{\mathrm{C}-}\right.$ $\mathrm{N}), 3110\left(v_{\mathrm{C}-\mathrm{H}}\right), 1322\left(v_{\mathrm{N}-\mathrm{N}}\right), 1521\left(\mathrm{vC}^{-\mathrm{NO}_{2}}\right),{ }^{1} \mathrm{H}$ NMR: $\delta(\mathrm{ppm}) 1.25\left(3 \mathrm{H}, \mathrm{t}, J=7.4 \mathrm{~Hz}, \mathrm{CH}_{3}\right)$, $3.07\left(2 \mathrm{H}, \mathrm{q}, J=7.4 \mathrm{~Hz}, \mathrm{CH}_{2}\right), 7.33-7.83(4 \mathrm{H}, \mathrm{m}$, $\mathrm{Ar}-\mathrm{H}), 9.41(1 \mathrm{H}, \mathrm{s}, \mathrm{N}=\mathrm{CH}),{ }^{13} \mathrm{C}$ NMR $\delta(\mathrm{ppm})$ 14.75 $\left(\mathrm{CH}_{3}\right.$ acyclic), $29.93\left(\mathrm{CH}_{2}\right.$ acyclic $)$, $158.71(\mathrm{~N}=\mathrm{CH}$ acyclic), 117.1-140.50 (C of aromatic ring $), \quad 156.4,159.6 \quad\left(\mathrm{C}_{2}, \mathrm{C}_{5} \quad\right.$ of thiadiazole ring), Anal. Calcd for: $\mathrm{C}_{11} \mathrm{H}_{10} \mathrm{~N}_{4} \mathrm{O}_{2} \mathrm{~S}$ : C, 50.37, H, $3.84 \mathrm{~N}, 21.36 \%$; found C, 50.32, $\mathrm{H}, 3.49, \mathrm{~N}, 21.14, \%$; Mass $262\left(\mathrm{M}^{+}\right)$.

\section{(4-nitro-benzylidene)-(5-ethyl-[1,3,4]}

thiadiazol-2-yl)-amine (1j) :m.p.176-178 ${ }^{0} \mathrm{C}$, yield $81 \%, \quad$ IR:1550 $\left(v_{\mathrm{C}-\mathrm{C}}\right), \quad 754 \quad\left(v_{\mathrm{C}-\mathrm{S}}\right)$, $1648\left(v_{\mathrm{N}=\mathrm{C}}\right), 1433\left(v_{\mathrm{C}-\mathrm{N}}\right), 3120\left(v_{\mathrm{C}-\mathrm{H}}\right), 1317\left(v_{\mathrm{N}-\mathrm{N}}\right)$, 1526( $\left(\mathrm{vC}-\mathrm{NO}_{2}\right) ;{ }^{1} \mathrm{H}$ NMR: $\delta(\mathrm{ppm}) 1.27(3 \mathrm{H}, \mathrm{t}, J$ $\left.=7.1 \mathrm{~Hz}, \mathrm{CH}_{3}\right), 3.08\left(2 \mathrm{H}, \mathrm{q}, J=7.1 \mathrm{~Hz} \mathrm{CH}_{2}\right)$, 7.39-7.95 (4H, m, Ar-H), $9.46(1 \mathrm{H}, \mathrm{s}, \mathrm{N}=\mathrm{CH})$; ${ }^{13} \mathrm{C}$ NMR $\delta(\mathrm{ppm}) 11.85\left(\mathrm{CH}_{3}\right.$ acyclic), 26.93
$\left(\mathrm{CH}_{2}\right.$ acyclic), 158.21(N=CH acyclic),117.1140.20 (C of aromatic ring), 157.7, $158.6\left(\mathrm{C}_{2}, \mathrm{C}_{5}\right.$ of thiadiazole ring). Anal. Calcd for: $\mathrm{C}_{11} \mathrm{H}_{10} \mathrm{~N}_{4}$ $\mathrm{O}_{2} \mathrm{~S}: \mathrm{C}, 50.37, \mathrm{H}, 3.84 \mathrm{~N}, 21.36 \%$; found $\mathrm{C}$, 50.22, H, 3.32, N, $21.21 \%$; Mass $262\left(\mathrm{M}^{+}\right)$.

\section{(2-methoxy-benzylidene)-(5-ethyl-[1,3,4]}

thiadiazol-2-yl)-amine(1k): m.p. $136-137^{\circ} \mathrm{C}$, yield $61 \%$, IR: $1549\left(v_{\mathrm{C}-\mathrm{C}}\right), 752\left(v_{\mathrm{C}-\mathrm{S}}\right), 1639\left(v_{\mathrm{N}=\mathrm{C}}\right)$, $1439\left(v_{\mathrm{C}-\mathrm{N}}\right), \quad 3090 \quad\left(v_{\mathrm{C}-\mathrm{H}}\right), \quad 1314\left(v_{\mathrm{N}-\mathrm{N}}\right)$, 2969( $\left(\mathrm{VOCH}_{3}\right) ;{ }^{1} \mathrm{H}$ NMR: $\delta$ (ppm)1.21 (3H, t, $J$ $\left.=7.1 \mathrm{~Hz} \mathrm{CH}_{3}\right), 3.17(2 \mathrm{H}, \mathrm{q}, J=7.1 \mathrm{~Hz} \mathrm{CH})^{2}$, $3.87\left(3 \mathrm{H}, \mathrm{s}, \mathrm{OCH}_{3}\right), 7.03-7.74(4 \mathrm{H}, \mathrm{m},) \mathrm{Ar}-\mathrm{H}$, 9.34 $(1 \mathrm{H}, \mathrm{s}, \mathrm{N}=\mathrm{CH}) ;{ }^{13} \mathrm{C}$ NMR $\delta(\mathrm{ppm}) 13.05$ $\left(\mathrm{CH}_{3}\right.$ acyclic $), \quad 28.93\left(\mathrm{CH}_{2}\right.$ acyclic $)$, $55.89\left(\mathrm{OCH}_{3}\right), 119.2-159.59$ ( $\mathrm{C}$ of aromatic ring), $159.52 \mathrm{~N}=\mathrm{CH}$ acyclic, $156.7,158.9\left(\mathrm{C}_{2}, \mathrm{C}_{5}\right.$ of thidiazole). Anal. Calcd for: $\mathrm{C}_{12} \mathrm{H}_{13} \mathrm{~N}_{3}$ OS: C,58.28, H,5 .30 N,16.99\%; found C, 58.12, H, 5.18, N, $16.91 \%$; Mass $247\left(\mathrm{M}^{+}\right)$.

\section{(3-methoxy-benzylidene)-(5-ethyl-[1,3,4]}

thiadiazol-2-yl)-amine (1l): m.p.134-136 ${ }^{\circ} \mathrm{C}$, yield 59\%, IR-1549 $\left(v_{\mathrm{C}-\mathrm{C}}\right), 755 \quad\left(v_{\mathrm{C}-\mathrm{S}}\right)$, $1651\left(v_{\mathrm{C}=\mathrm{N}}\right), 1440\left(v_{\mathrm{C}-\mathrm{N}}\right), 3085\left(v_{\mathrm{C}-\mathrm{H}}\right), 1314\left(v_{\mathrm{N}-\mathrm{N}}\right)$, $2973\left(\mathrm{vOCH}_{3}\right) ;{ }^{1} \mathrm{H}$ NMR: $\delta 1.19(\mathrm{ppm})(3 \mathrm{H}, \mathrm{t}, J$ $=7.0 \mathrm{~Hz} \mathrm{CH}$ ), $2.97(2 \mathrm{H}, \mathrm{q}, J=7.0 \mathrm{~Hz} \mathrm{CH})$, $3.77\left(3 \mathrm{H}, \mathrm{s}, \mathrm{OCH}_{3}\right), 7.06-7.81(4 \mathrm{H}, \mathrm{m}, \mathrm{Ar}-\mathrm{H})$ $9.41(1 \mathrm{H}, \mathrm{s}, \mathrm{N}=\mathrm{CH}),{ }^{13} \mathrm{C}$ NMR $\delta(\mathrm{ppm}) 11.95$ $\left(\mathrm{CH}_{3}\right.$ acyclic $), \quad 26.91\left(\mathrm{CH}_{2}\right.$ acyclic $), \quad 55.79$ $\left(\mathrm{OCH}_{3}\right), 111.02-157.69$ (C of aromatic ring), $158.71(\mathrm{~N}=\mathrm{CH}$ acyclic $), 156.5,159.4\left(\mathrm{C}_{2}, \mathrm{C}_{5}\right.$ of thiadiazole). Anal. Calcd for: $\mathrm{C}_{12} \mathrm{H}_{13} \quad \mathrm{~N}_{3} \mathrm{OS}$ :C,58.28, H,5.30 N,16.99 \%; found C, 58.12, $\mathrm{H}, 16, \mathrm{~N}, 16.81 \%$; Mass $247\left(\mathrm{M}^{+}\right)$.

\section{(4-methoxy-benzylidene)-(5-ethyl-[1,3,4}

Jthiadiazol-2-yl)-amine (1m): m.p.136-138 ${ }^{\circ} \mathrm{C}$, yield 57\%, IR-1549( $\left.v_{\mathrm{C}-\mathrm{C}}\right), 755\left(v_{\mathrm{C}-\mathrm{S}}\right), 1628\left(v_{\mathrm{C}=\mathrm{N}}\right)$, $1441\left(v_{\mathrm{C}-\mathrm{N}}\right), \quad 3095 \quad\left(v_{\mathrm{C}-\mathrm{H}}\right), 1314\left(v_{\mathrm{N}-\mathrm{N}}\right), \quad 1109$ $\left(\mathrm{vCOCH}_{3}\right) ;{ }^{1} \mathrm{H}$ NMR: $\delta 1.26(\mathrm{ppm})(3 \mathrm{H}, \mathrm{t}, J=$ $\left.7.1 \mathrm{~Hz}, \mathrm{CH}_{3}\right), 3.17\left(2 \mathrm{H}, \mathrm{q}, J=7.1 \mathrm{~Hz} \mathrm{CH}_{2}\right), 3.84$ $\left(3 \mathrm{H}, \mathrm{s}, \quad \mathrm{OCH}_{3}\right), 7.26-7.73(4 \mathrm{H}, \mathrm{m}, \mathrm{Ar}-\mathrm{H}), 9.35$ 
$(1 \mathrm{H}, \mathrm{s}, \mathrm{N}=\mathrm{CH}) ;{ }^{13} \mathrm{C}$ NMR: $\delta(\mathrm{ppm}) 11.95\left(\mathrm{CH}_{3}\right.$ acyclic), 26.98 $\left(\mathrm{CH}_{2}\right.$ acyclic $), \quad 55.51\left(\mathrm{OCH}_{3}\right)$, 114.5-160.40 (C of aromatic ring), $158.61(\mathrm{~N}=\mathrm{CH}$ acyclic $), 160.4,156.7\left(\mathrm{C}_{2}, \mathrm{C}_{5}\right.$ of thiadiazole). Anal. Calcd for: $\mathrm{C}_{12} \mathrm{H}_{13} \mathrm{~N}_{3}$ OS : C, 58.28, H, $5.30 \mathrm{~N}, 16.99 \%$; found C, 58.12, H, $5.21, \mathrm{~N}, 16.11 \%$; Mass $247\left(\mathrm{M}^{+}\right)$.

\section{Synthesis of 3-(5-ethyl-[1,3,4]thiadiazole-2-yl)- 2-phenyl-thiazolidin-4-one (2a):}

Equimolar solution of the compound 1a $(0.004$ mole) and mercaptoacetic acid (0.004 mole) in methanol $(30 \mathrm{~mL})$ and a pinch of anhydrous $\mathrm{ZnCl}_{2}$ as a catalyst was first continuously stirred on a magnetic stirrer for about $02 \mathrm{hr}$ followed by refluxing for about $11 \mathrm{hrs}$ on a steam bath at $85-95^{\circ} \mathrm{C}$ temperature. After the completion of the reaction the excess of methanol was removed and the product thus obtained was washed with water and purified over a silica gel column and the product recrystallized from ethanol to yield compound 2a, yellow crystalline

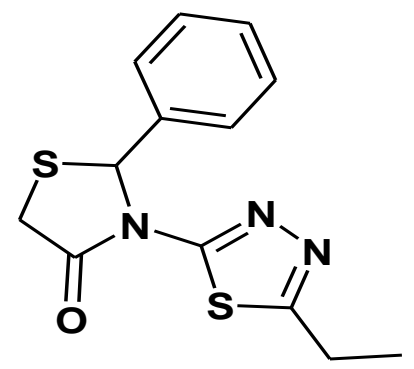

Compoun-2a

compound. m.p.190-192 ${ }^{\circ} \mathrm{C}$, yield $75 \%$, IR$1453\left(v_{\mathrm{C}-\mathrm{C}}\right), 1590\left(v_{\mathrm{C}=\mathrm{C}}\right), 1310\left(v_{\mathrm{N}-\mathrm{N}}\right), 1670\left(v_{\mathrm{C}=\mathrm{N}}\right)$, $712\left(v_{\mathrm{C}-\mathrm{S}}\right), 1710\left(v_{\mathrm{C}=\mathrm{O}}\right)$ 2949( $\left(v_{\mathrm{C}-\mathrm{H}}\right) ;{ }^{1} \mathrm{H}$ NMR: $\delta(\mathrm{ppm}) 1.09(3 \mathrm{H}, \mathrm{t}, J=7.3 \mathrm{~Hz} \mathrm{CH}), 1.04(2 \mathrm{H}$, q, $\left.J=7.3 \mathrm{~Hz} \mathrm{CC_{2 }}\right), 3.66(2 \mathrm{H}, \mathrm{d}, J=15.8 \mathrm{~Hz}$ $\mathrm{CH}$, thiazolidinone $), \quad 6.13 \quad(1 \mathrm{H}, \quad \mathrm{s}) \quad \mathrm{CH}$ thiazolidinone, 7.32-7.48 (5H, m, Ar- $\mathrm{H}) ;{ }^{13} \mathrm{C}$ NMR: $\delta(\mathrm{ppm}) 13.25\left(\mathrm{CH}_{3}\right.$ acyclic), $29.13\left(\mathrm{CH}_{2}\right.$ acyclic), 128.2-129.2(C of aromatic ring), 157.8,161.3 $\left(\mathrm{C}_{2}, \mathrm{C}_{5}\right.$ of thiadiazole ring $)$,
170.6(C $=\mathrm{O}$ cyclic thiazolidinone $), 34.5\left(\mathrm{CH}_{2}\right.$ thiazolidinone), 69.7(CH cyclic thiazolidinone); Anal. Calcd for: $\mathrm{C}_{13} \mathrm{H}_{13} \mathrm{~N}_{3} \mathrm{OS}_{2}: \mathrm{C}, 53.58, \mathrm{H}$, $4.50, \mathrm{~N}, 14.42 \%$; found $\mathrm{C}, 53.41, \mathrm{H}, 4.11, \mathrm{~N}$, $14.21 \%$; Mass 291( $\left.\mathrm{M}^{+}\right)$.

The compounds $\mathbf{2 b}-\mathbf{2} \mathbf{m}$ were synthesized by the similar method as described for $\mathbf{2 a}$.

\section{2-(2-chloro-phenyl)-3-(5-ethyl-[1,3,4] \\ thiadiazole-2-yl)-thiazolidin-4-one (2b):}

m.p. $175-177^{\circ} \mathrm{C}$, yield $65 \%$, IR: $1451\left(v_{\mathrm{C}-\mathrm{C}}\right)$, $1550\left(v_{\mathrm{C}=\mathrm{C}}\right), 1308\left(v_{\mathrm{N}-\mathrm{N}}\right), 1627\left(v_{\mathrm{C}=\mathrm{N}}\right), 711\left(v_{\mathrm{C}-\mathrm{S}}\right)$, $1712\left(v_{\mathrm{C}=\mathrm{O}}\right) 720\left(v_{\mathrm{C}-\mathrm{Cl}}\right) ;{ }^{1} \mathrm{H}$ NMR: $\delta(\mathrm{ppm}) 1.09$ $\left(3 \mathrm{H}, \mathrm{t}, J=7.3 \mathrm{~Hz}, \mathrm{CH}_{3}\right), 2.87(2 \mathrm{H}, \mathrm{q}, J=7.3$ $\left.\mathrm{Hz}, \mathrm{CH}_{2}\right), 3.67(2 \mathrm{H} \mathrm{d}, J=15.8 \mathrm{~Hz} \mathrm{~S}-\mathrm{CH}$ thiazolidinone), $6.12(1 \mathrm{H}, \mathrm{s}, \mathrm{CH}$ thiazolidinone), 7.21-7.64 (4H, m, Ar-H); ${ }^{13} \mathrm{C} \quad \mathrm{NMR} \quad \delta$ (ppm)12.15( $\mathrm{CH}_{3}$ acyclic), $29.98\left(\mathrm{CH}_{2}\right.$ acyclic), 127.6-133.2(C of aromatic ring), 161.3, $156.8\left(\mathrm{C}_{2}, \mathrm{C}_{5}\right.$ of thiadiazole ring $) .171 .5(\mathrm{C}=\mathrm{O}$ cyclic thiazolidinone), $34.5\left(\mathrm{CH}_{2}\right.$ thiazolidinone), 69.0(CH cyclic thiazolidinone). Anal. Calcd for : $\mathrm{C}_{13} \mathrm{H}_{12} \mathrm{Cl} \mathrm{N} \mathrm{OS}_{2}: \quad \mathrm{C}, 47.92, \mathrm{H}, 3.71, \mathrm{~N}, 12.90$ $\%$; found $\mathrm{C}, 46.91, \mathrm{H}, 3.01, \mathrm{~N}, 11.71 \%$; Mass $325\left(\mathrm{M}^{+}\right)$.

\section{2-(3-chloro-phenyl)-3-(5-ethyl-[1,3,4] thiadiazole-2-yl)-thiazolidin-4-one (2c):}

m.p. $172-175^{\circ} \mathrm{C}$, yield $55 \%$, IR:1450( $\left.v_{\mathrm{C}-\mathrm{C}}\right), 1555$ $\left(v_{\mathrm{C}=\mathrm{C}}\right), 1316\left(v_{\mathrm{N}-\mathrm{N}}\right), 1629\left(v_{\mathrm{C}=\mathrm{N}}\right), 712\left(v_{\mathrm{C}-\mathrm{S}}\right), 1714$ $\left(v_{\mathrm{C}=0}\right), 730\left(v_{\mathrm{C}-\mathrm{Cl}}\right) ;{ }^{1} \mathrm{H}$ NMR: $\delta(\mathrm{ppm}) 1.02(3 \mathrm{H}, \mathrm{t}$, $\left.J=7.3 \mathrm{~Hz}, \mathrm{CH}_{3}\right), 2.88\left(2 \mathrm{H}, \mathrm{q}, J=7.3 \mathrm{~Hz}, \mathrm{CH}_{2}\right)$, $3.61(2 \mathrm{H}, \mathrm{d}, J=15.9 \mathrm{~Hz}, \mathrm{~S}-\mathrm{CH}$ thiazolidinone), $6.18(1 \mathrm{H}, \mathrm{s}, \mathrm{CH}$ thiazolidinone), 7.22-7.40 $(4 \mathrm{H}$, $\mathrm{m}, \quad \mathrm{Ar}-\mathrm{H}) ;{ }^{13} \mathrm{C} \quad \mathrm{NMR} \quad: \delta \quad(\mathrm{ppm}) 13.39\left(\mathrm{CH}_{3}\right.$ acyclic), $28.23\left(\mathrm{CH}_{2}\right.$ acyclic), 127.6-137.0 (C of aromatic ring $), 160.3,157.2\left(\mathrm{C}_{2}, \mathrm{C}_{5}\right.$ of thidiazole ring), 179.8 ( $\mathrm{C}=\mathrm{O}$ cyclic thiazolidinone), 34.8 $\left(\mathrm{CH}_{2} \quad\right.$ thiazolidinone $), \quad 69.6 \quad(\mathrm{CH} \quad$ cyclic thiazolidinone); Anal. Calcd. for: $\mathrm{C}_{13} \mathrm{H}_{12} \mathrm{Cl} \mathrm{N}$ $\mathrm{OS}_{2} \mathrm{C}, 47.92, \mathrm{H}, 3.71, \mathrm{~N}, 12.90 \%$; found $\mathrm{C}$, 47.71, H, 3.43, N, $12.51 \%$; Mass $325\left(\mathrm{M}^{+}\right)$. 
2-(4-chloro-phenyl)-3-(5-ethyl-[1,3,4]

thiadiazole-2-yl)-thiazolidin-4-one (2d):

m.p. $173-176^{\circ} \mathrm{C}$, yield $59 \%$, IR:1450 ( $\left.v_{\mathrm{C}-\mathrm{C}}\right)$, $1542\left(v_{\mathrm{C}=\mathrm{C}}\right), 1315\left(v_{\mathrm{N}-\mathrm{N}}\right), 1630\left(v_{\mathrm{C}=\mathrm{N}}\right), 711\left(v_{\mathrm{C}-\mathrm{S}}\right)$, $1720\left(v_{\mathrm{C}=\mathrm{O}}\right), 730\left(v_{\mathrm{C}-\mathrm{Cl}}\right) ;{ }^{1} \mathrm{H}$ NMR: $\delta(\mathrm{ppm}) 1.07$ $(3 \mathrm{H}, \mathrm{t}, J=7.3 \mathrm{~Hz} \mathrm{CH}), 2.88(2 \mathrm{H}, \mathrm{q}, J=7.3 \mathrm{~Hz}$ $\left.\mathrm{CH}_{2}\right), 3.70(2 \mathrm{H}, \mathrm{d}, J=15.8 \mathrm{~Hz}, \mathrm{~S}-\mathrm{CH}$ thiazolidinone, $) \quad 6.19 \quad(1 \mathrm{H}, \quad \mathrm{s}, \quad \mathrm{CH}$ thiazolidinone), 7.54-7.56 (4H, m, Ar-H); ${ }^{13} \mathrm{C}$ NMR: $\delta(\mathrm{ppm}) 129.5-139.3(\mathrm{C}$ of aromatic ring), 12.75( $\mathrm{CH}_{3}$ acyclic $), 27.93\left(\mathrm{CH}_{2}\right.$ acyclic $)$, 34.5( $\left(\mathrm{CH}_{2}\right.$ thiazolidinone), $67.9(\mathrm{CH}$ cyclic thiazolidinone $) \quad 159.4, \quad 156.8 \quad\left(\mathrm{C}_{2}, \mathrm{C}_{5}\right.$ of thiadiazole ring), $171.6(\mathrm{C}=\mathrm{O}$ thiazolidinone $)$; Anal. Calcd For: $\mathrm{C}_{13} \mathrm{H}_{12} \mathrm{Cl} \mathrm{N}_{3} \mathrm{OS}_{2}$ : C, 47.92, H, $3.71, \mathrm{~N}, 12.90 \%$; found $\mathrm{C}, 47.51, \mathrm{H}, 3.43, \mathrm{~N}$, $12.81 \%$; Mass 325( $\left.\mathrm{M}^{+}\right)$.

\section{2-(2-bromo-phenyl)-3-(5-ethyl-[1,3,4] thiadiazole-2-yl)-thiazolidin-4-one (2e):}

m.p. $180-182^{\circ} \mathrm{C}$, yield $69 \%$, IR: $1446\left(v_{\mathrm{C}-\mathrm{C}}\right)$, $1545\left(v_{\mathrm{C}=\mathrm{C}}\right), 1318\left(v_{\mathrm{N}-\mathrm{N}}\right), 1630\left(v_{\mathrm{C}=\mathrm{N}}\right), 714\left(v_{\mathrm{C}-\mathrm{S}}\right)$, $1719\left(v_{\mathrm{C}=\mathrm{O}}\right), 545\left(v_{\mathrm{C}-\mathrm{Br}}\right) ;{ }^{1} \mathrm{H}$ NMR: $\delta 1.06(3 \mathrm{H}, \mathrm{t}$, $\left.J=7.3 \mathrm{~Hz}, \mathrm{CH}_{3}\right), 2.87\left(2 \mathrm{H}, \mathrm{q}, J=7.3 \mathrm{~Hz}, \mathrm{CH}_{2}\right)$, $3.69(2 \mathrm{H}, \mathrm{d}, J=15.9 \mathrm{~Hz}) \mathrm{S}-\mathrm{CH}$ thiazolidinone, $6.13(1 \mathrm{H}, \mathrm{s}, \mathrm{CH}$ thiazolidinone), 7.03-7.48 $(4 \mathrm{H}$, $\mathrm{m}, \mathrm{Ar}-\mathrm{H}) ;{ }^{13} \mathrm{C}$ NMR $\delta$ (ppm)-120.7.2-132.7 (C of aromatic ring), $12.55\left(\mathrm{CH}_{3}\right.$ acyclic $), 27.23$ $\left(\mathrm{CH}_{2}\right.$ acyclic $), 160.3,155.3\left(\mathrm{C}_{2}, \mathrm{C}_{5}\right.$ of thiadiazole ring), $170.2(\mathrm{C}=\mathrm{O}$ cyclic thiazolidinone), 34.2 $\left(\mathrm{CH}_{2} \quad\right.$ thiazolidinone), $68.5(\mathrm{CH}$ cyclic thiazolidinone); Anal. Calcd for: $\mathrm{C}_{13} \mathrm{H}_{12} \mathrm{Br} \mathrm{N}$ $\mathrm{OS}_{2}$ : C, 42.17, H, 3.27, N, $11.35 \%$; found C, 42.01, H, 3.11, N, $11.01 \%$; Mass 370 $\left(\mathrm{M}^{+}\right)$.

\section{2-(3-bromo-phenyl)-3-(5-ethyl-[1,3,4] thiadiazole-2-yl)-thiazolidin-4-one (2f):}

m.p. $181-184^{0} \mathrm{C}$, yield $65 \%$, IR:1448 ( $\left.v_{\mathrm{C}-\mathrm{C}}\right)$, $1540\left(v_{\mathrm{C}=\mathrm{C}}\right), 1313\left(v_{\mathrm{N}-\mathrm{N}}\right), 1630\left(v_{\mathrm{C}=\mathrm{N}}\right), 715\left(v_{\mathrm{C}-\mathrm{S}}\right)$, $1712\left(v_{\mathrm{C}=\mathrm{O}}\right), 525\left(v_{\mathrm{C}-\mathrm{Br}}\right) ;{ }^{1} \mathrm{H}$ NMR: $\delta(\mathrm{ppm})$ $1.10\left(3 \mathrm{H}, \mathrm{t}, J=7.3 \mathrm{~Hz}, \mathrm{CH}_{3}\right), 2.78(2 \mathrm{H}, \mathrm{q}, J=$ $\left.7.3 \mathrm{~Hz}, \mathrm{CH}_{2}\right), 3.70(2 \mathrm{H}, \mathrm{d}, J=15.9 \mathrm{~Hz}, \mathrm{~S}-\mathrm{CH}$ thiazolidinone), $\quad 6.15 \quad(1 \mathrm{H}, \mathrm{s}, \quad \mathrm{CH}$ thiazolidinone), 7.04-7.35 (4H, m, Ar-H); ${ }^{13} \mathrm{C}$ NMR: $\delta$ (ppm) 118.2-137.0 (C of aromatic ring), $12.76\left(\mathrm{CH}_{3}\right.$ acyclic $), 27.83\left(\mathrm{CH}_{2}\right.$ acyclic $), 158.5$, $157.4\left(\mathrm{C}_{2}, \mathrm{C}_{5}\right.$ of thiadiazole ring $), 168.9(\mathrm{C}=\mathrm{O}$ cyclic thiazolidinone), $34.4 \quad\left(\mathrm{CH}_{2}\right.$ thiazolidinone), 69.1(cyclic $\mathrm{CH}$ thiazolidinone); Anal. Calcd for: $\mathrm{C}_{13} \mathrm{H}_{12} \mathrm{Br} \mathrm{N}_{3} \mathrm{OS}_{2}$ : C, 42.17, H,3. 27, N, $11.35 \%$; found C, 42.11, H, 3.21, N, $11.01 \%$; Mass $370\left(\mathrm{M}^{+}\right)$.

\section{2-(4-bromo-phenyl)-3-(5-ethyl-[1,3,4] thiadiazole-2-yl)-thiazolidin-4-one (2g):}

m.p. $182-183^{\circ} \mathrm{C}$, yield $67 \%$, IR:1441 $\left(v_{\mathrm{C}-\mathrm{C}}\right)$, $1542\left(v_{\mathrm{C}=\mathrm{C}}\right), 1314\left(v_{\mathrm{N}-\mathrm{N}}\right), 1629\left(v_{\mathrm{C}=\mathrm{N}}\right), 712\left(v_{\mathrm{C}-\mathrm{S}}\right)$, $1713\left(v_{\mathrm{C}=\mathrm{O}}\right), 542\left(v_{\mathrm{C}-\mathrm{Br}}\right) ;{ }^{1} \mathrm{H}$ NMR: $\delta(\mathrm{ppm}) 1.09$ $\left(3 \mathrm{H}, \mathrm{t}, J=7.3 \mathrm{~Hz}, \mathrm{CH}_{3}\right), 2.78(2 \mathrm{H}, \mathrm{q}, J=7.3 \mathrm{~Hz}$, $\left.\mathrm{CH}_{2}\right), 3.69(2 \mathrm{H}, \mathrm{d}, \quad J=15.9 \mathrm{~Hz}, \mathrm{~S}-\mathrm{CH}$ thiazolidinone), $6.16(1 \mathrm{H}, \mathrm{s}, \mathrm{CH}$ thiazolidinone), 7.30-7.63 (4H, m, Ar-H); ${ }^{13} \mathrm{C}$ NMR: $\delta$ (ppm) $13.41\left(\mathrm{CH}_{3}\right.$ acyclic), $28.23\left(\mathrm{CH}_{2}\right.$ acyclic $), 124.2-$ 139.3 (C of aromatic ring), 169.2, $155.7\left(\mathrm{C}_{2}, \mathrm{C}_{5}\right.$ of thiadiazole ring $), \quad 169.5 \quad(\mathrm{C}=\mathrm{O}$ cyclic thiazolidinone), $33.5\left(\mathrm{CH}_{2}\right.$ thiazolidinone), 68.4 (cyclic $\mathrm{CH}$ thiazolidinone); Anal. Calcd for : $\mathrm{C}_{13} \mathrm{H}_{12} \mathrm{Br} \mathrm{N}_{3} \mathrm{OS}_{2}$ : C, 42.17, H,3.27, N,11.35\%; found $\mathrm{C}, 42.01, \mathrm{H}, 3.20, \mathrm{~N}, 11.01 \%$; Mass 370 $\left(\mathrm{M}^{+}\right)$.

\section{2-(2-nitro-phenyl)-3-(5-ethyl-[1,3,4] thiadiazole-2-yl)-thiazolidin-4-one $(2 \mathrm{~h})$ :}

m.p. $176-179^{\circ} \mathrm{C}$, yield $65 \%$, IR:1446 ( $\left.v_{\mathrm{C}-\mathrm{C}}\right)$, $1539\left(v_{\mathrm{C}=\mathrm{C}}\right), 1316\left(v_{\mathrm{N}-\mathrm{N}}\right), 1630\left(v_{\mathrm{C}=\mathrm{N}}\right), 720\left(v_{\mathrm{C}-\mathrm{S}}\right)$, $1716\left(v_{\mathrm{C}=\mathrm{O}}\right), 1526\left(v \mathrm{C}-\mathrm{NO}_{2}\right) ;{ }^{1} \mathrm{H}$ NMR: $\delta(\mathrm{ppm})$ $1.15\left(3 \mathrm{H}, \mathrm{t}, J=7.3 \mathrm{~Hz}, \mathrm{CH}_{3}\right), 2.81(2 \mathrm{H}, \mathrm{q}, J=$ $\left.7.3 \mathrm{~Hz}, \mathrm{CH}_{2}\right), 3.71(2 \mathrm{H}, \mathrm{d}, J=15.9 \mathrm{~Hz}, \mathrm{CH}$ thiazolidinone), $6.14(1 \mathrm{H}, \mathrm{CH}$ thiazolidinone, $\mathrm{s})$, 7.50-8.08 (4H, m,) Ar-H; ${ }^{13} \mathrm{C}$ NMR : $\delta$ (ppm) $13.05\left(\mathrm{CH}_{3}\right.$ acyclic $), 28.83\left(\mathrm{CH}_{2}\right.$ acyclic $), 127.6-$ 148.4 (C of aromatic ring), 161.3, $156.8\left(\mathrm{C}_{2}, \mathrm{C}_{5}\right.$ of thiadiazole ring $), \quad 170.9 \quad(\mathrm{C}=\mathrm{O} \quad$ cyclic thiazolidinone), $34.4\left(\mathrm{CH}_{2}\right.$ thiazolidinone), 69.2 (CH cyclic thiazolidinone); Anal. Calcd for: $\mathrm{C}_{13} \mathrm{H}_{12} \mathrm{~N}_{4} \mathrm{O}_{3} \mathrm{~S}_{2}: \mathrm{C}, 46.42, \mathrm{H}, 3.60, \mathrm{~N}, 16.66$, O, 
14.27, S, $19.06 \%$; found C, 45.21, H, 3.20, N, $15.80 \%$; Mass $336\left(\mathrm{M}^{+}\right)$.

\section{2-(3-nitro-phenyl)-3-(5-ethyl-[1,3,4] \\ thiadiazole-2-yl)-thiazolidin-4-one (2i):}

m.p. $175-177^{0} \mathrm{C}$, yield $85 \%$, IR:1441 ( $\left.v_{\mathrm{C}-\mathrm{C}}\right)$, $1539\left(v_{\mathrm{C}=\mathrm{C}}\right), 1311\left(v_{\mathrm{N}-\mathrm{N}}\right), 1630\left(v_{\mathrm{C}=\mathrm{N}}\right), 720\left(v_{\mathrm{C}-\mathrm{S}}\right)$, $1715\left(v_{\mathrm{C}=\mathrm{O}}\right), 1528\left(v_{\mathrm{C}-\mathrm{NO}_{2}}\right) ;{ }^{1} \mathrm{H} \mathrm{NMR}: \delta(\mathrm{ppm})$ $1.08\left(3 \mathrm{H}, \mathrm{t}, J=7.3 \mathrm{~Hz}, \mathrm{CH}_{3}\right), 2.81(2 \mathrm{H}, \mathrm{q}, \quad J=$ $7.3 \mathrm{~Hz} \mathrm{CH}$ ), $3.76(2 \mathrm{H}, \mathrm{d}, J=15.9 \mathrm{~Hz}, \mathrm{CH}$ thiazolidin-4-one), $6.31(1 \mathrm{H}, \mathrm{s}, \mathrm{CH}$ thiazolidin4-one,) 7.54-8.04 (4H, m, Ar-H); ${ }^{13} \mathrm{C}$ NMR: $\delta$ (ppm) $13.35\left(\mathrm{CH}_{3}\right.$ acyclic), $28.97\left(\mathrm{CH}_{2}\right.$ acyclic $)$, 117.3-140.5(C of aromatic ring), 159.4-158.2 $\left(\mathrm{C}_{2}, \mathrm{C}_{5}\right.$ of thiadiazole $), 171.6 \quad \mathrm{C}=\mathrm{O}$ Cyclic thiazolidinone), $34.4\left(\mathrm{CH}_{2}\right.$ thiazolidinone $), 68.8$ ( $\mathrm{CH}$ cyclic thiazolidinone); Anal. Calcd for: $\mathrm{C}_{13} \mathrm{H}_{12} \mathrm{~N}_{4} \mathrm{O}_{3} \mathrm{~S}_{2}$ : C, 46.42, H, 3. 60, N, $16.66 \%$; found C, 46.11, H, 3.18, N, $16.27 \%$; Mass 336 . $\left(\mathrm{M}^{+}\right)$.

\section{2-(4-nitro-phenyl)-3-(5-ethyl-[1,3,4] thiadiazole-2-yl)-thiazolidin-4-one (2j):}

m.p. $176-179^{\circ} \mathrm{C}$, yield $83 \%$, IR:1442 $\left(v_{\mathrm{C}-\mathrm{C}}\right)$, $1546\left(v_{\mathrm{C}=\mathrm{C}}\right), 1319\left(v_{\mathrm{N}-\mathrm{N}}\right), 1630\left(v_{\mathrm{C}=\mathrm{N}}\right), 720\left(v_{\mathrm{C}-\mathrm{S}}\right)$, $1717\left(v_{\mathrm{C}=\mathrm{O}}\right),, 1539\left(v \mathrm{C}-\mathrm{NO}_{2}\right) ;{ }^{1} \mathrm{H}$ NMR: $\delta(\mathrm{ppm})$ $1.11\left(3 \mathrm{H}, \mathrm{t}, J=7.3 \mathrm{~Hz}, \mathrm{CH}_{3}\right), 2.84(2 \mathrm{H}, \mathrm{q}, J=$ $\left.7.3 \mathrm{~Hz}, \mathrm{CH}_{2}\right), 3.76(2 \mathrm{H}, \mathrm{d}, J=15.8 \mathrm{~Hz}, \mathrm{CH}$ thiazolidinone), $6.09(1 \mathrm{H}, \mathrm{s}, \mathrm{CH}$ thiazolidinone), 7.42-8.08 (4H, m, Ar-H, $) ;{ }^{13} \mathrm{C} \quad \mathrm{NMR} \delta$ (ppm)12.76( $\mathrm{CH}_{3}$ acyclic), $27.93\left(\mathrm{CH}_{2}\right.$ acyclic $)$, 117.3-140.5(C of aromatic ring), 160.4,157.8 $\left(\mathrm{C}_{2}, \mathrm{C}_{5}\right.$ of thiadiazole ring $), 171.5(\mathrm{C}=\mathrm{O}$ Cyclic thiazolidinone), $35.4\left(\mathrm{CH}_{2}\right.$ thiazolidinone), 67.9( $\mathrm{CH}$ cyclic thiazolidinone); Anal. Calcd for: $\mathrm{C}_{13} \mathrm{H}_{12} \mathrm{~N}_{4} \mathrm{O}_{3} \mathrm{~S}_{2}$ : C, 46.42, H,3. 60, N, $16.66 \%$; found $\mathrm{C}, 46.31, \mathrm{H}, 3.24, \mathrm{~N}, 16.51 \%$; Mass 336 $\left(\mathrm{M}^{+}\right)$.

\section{2-(2-methoxy-phenyl)-3-(5-ethyl-[1,3,4] thiadiazole-2-yl)-thiazolidin-4-one (2k):}

m.p. $135-137^{0} \mathrm{C}$, yield $60 \%$, IR: $1449\left(v_{\mathrm{C}-\mathrm{C}}\right)$, $1544\left(v_{\mathrm{C}=\mathrm{C}}\right), 1317\left(v_{\mathrm{N}-\mathrm{N}}\right), 1629\left(v_{\mathrm{C}=\mathrm{N}}\right), 723\left(v_{\mathrm{C}-\mathrm{S}}\right)$, $1711\left(v_{\mathrm{C}=\mathrm{O}}\right) 2970\left(v_{\mathrm{OCH}_{3}}\right) ;{ }^{1} \mathrm{H}$ NMR: $\delta 1.08$ $\left(3 \mathrm{H}, \mathrm{t}, \mathrm{J}=7.3 \mathrm{~Hz}, \mathrm{CH}_{3}\right), 2.78(2 \mathrm{H}, \mathrm{q}, \mathrm{J}=7.3 \mathrm{~Hz}$, $\left.\mathrm{CH}_{2}\right), 3.71(2 \mathrm{H}, \quad \mathrm{d}, \quad \mathrm{J}=15.9 \mathrm{~Hz}, \mathrm{CH}$ thiazolidinone), $3.79\left(3 \mathrm{H}, \mathrm{s}, \mathrm{OCH}_{3}\right), 6.03(1 \mathrm{H}, \mathrm{s}$, $\mathrm{CH}$ thiazolidinone), 6.89-7.37 (4H, m, Ar-H,); ${ }^{13} \mathrm{C} \mathrm{NMR}: \delta$ (ppm) $12.25\left(\mathrm{CH}_{3}\right.$ acyclic $), 27.93$ $\left(\mathrm{CH}_{2}\right.$ acyclic), 55.4( $\left(\mathrm{OCH}_{3}\right), 113.7-155.8$ ( $\mathrm{C}$ of aromatic ring $), 161.3,156.8\left(\mathrm{C}_{2}, \mathrm{C}_{5}\right.$ of thiadiazole ring).172.5 ( $\mathrm{C}=\mathrm{O}$ cyclic thiazolidinone $), 34.4$ $\left(\mathrm{CH}_{2} \quad\right.$ thiazolidinone $), \quad 69.7(\mathrm{CH} \quad$ cyclic thiazolidinone); Anal. Calcd for: $\mathrm{C}_{14} \mathrm{H}_{15} \mathrm{~N}_{4} \mathrm{O}_{2} \mathrm{~S}_{2}$ C, 52.32, H,4. 70, N, $13.07 \%$; found C, 52.11, $\mathrm{H}, 4.24, \mathrm{~N}, 13.00 \%$; Mass $321\left(\mathrm{M}^{+}\right)$.

\section{2-(3-methoxy-phenyl)-3-(5-ethyl-[1,3,4] thiadiazole-2-yl)-thiazolidin-4-one (2l):}

m.p.134-1136 ${ }^{0} \mathrm{C}$, yield $58 \%$, IR - $1441\left(v_{\mathrm{C}-\mathrm{C}}\right)$, $1542\left(v_{\mathrm{C}=\mathrm{C}}\right), 1313\left(v_{\mathrm{N}-\mathrm{N}}\right), 1630\left(v_{\mathrm{C}=\mathrm{N}}\right), 706\left(v_{\mathrm{C}-\mathrm{S}}\right)$, $1712\left(v_{\mathrm{C}=\mathrm{O}}\right) 2971\left(v_{\mathrm{OCH}_{3}}\right) ;{ }^{1} \mathrm{H}$ NMR: $\delta(\mathrm{ppm})$ $1.11\left(3 \mathrm{H}, \mathrm{t}, J=7.3 \mathrm{~Hz}, \mathrm{CH}_{3}\right), 2.79(2 \mathrm{H}, \mathrm{q}, J=$ $\left.7.3 \mathrm{~Hz}, \mathrm{CH}_{2}\right), 3.76(2 \mathrm{H}, \mathrm{d}, J=15.8 \mathrm{~Hz}, \mathrm{CH}$ thiazolidin-4-one), $3.73\left(3 \mathrm{H}, \mathrm{s}, \mathrm{OCH}_{3}\right), 6.26$ (1H, s CH thiazolidin-4-one), 6.88-7.24 (4H, m, Ar-H); ${ }^{13} \mathrm{C}$ NMR $: \delta(p p m) \quad 13.15\left(\mathrm{CH}_{3}\right.$ acyclic), 29.13 $\left(\mathrm{CH}_{2}\right.$ acyclic), 55.7 $\left(\mathrm{OCH}_{3}\right), 111.7-157.8(\mathrm{C}$ of aromatic ring), 169.3, $115.8 \quad\left(\mathrm{C}_{2}, \mathrm{C}_{5}\right.$ of thiadiazole ring $), \quad 172.2 \quad(\mathrm{C}=\mathrm{O} \quad$ cyclic thiazolidinone), $34.4 \quad\left(\mathrm{CH}_{2}\right.$ thiazolidinone $)$, 68.7(cyclic $\mathrm{CH}$ thiazolidinone); Anal. Calcd for: $\mathrm{C}_{14} \mathrm{H}_{15} \mathrm{~N}_{4} \mathrm{O}_{2} \mathrm{~S}_{2}: \mathrm{C}, 52.32, \mathrm{H}, 4.70, \mathrm{~N}, 13.07 \%$; found $\mathrm{C}, 52.21, \mathrm{H}, 4.14, \mathrm{~N}, 13.00 \%$; Mass 321 $\left(\mathrm{M}^{+}\right)$.

\section{2-(4-methoxy-phenyl)-3-(5-ethyl-[1,3,4] thiadiazole-2-yl)-thiazolidin-4-one (2m):}

m.p.136- $137^{\circ} \mathrm{C}$, yield $55 \%$, IR: $1443\left(v_{\mathrm{C}-\mathrm{C}}\right)$, $1565\left(v_{\mathrm{C}=\mathrm{C}}\right), 1316\left(v_{\mathrm{N}-\mathrm{N}}\right), 1632\left(v_{\mathrm{C}=\mathrm{N}}\right), 707\left(v_{\mathrm{C}-\mathrm{S}}\right)$, $1710\left(v_{\mathrm{C}=\mathrm{O}}\right) 2968\left(v \mathrm{OCH}_{3}\right) ;{ }^{1} \mathrm{H}$ NMR: $\delta$ (ppm)1.17 (3H, t, $\left.J=7.3 \mathrm{~Hz}, \mathrm{CH}_{3}\right), 2.83(2 \mathrm{H}, \mathrm{q}$, $\left.J=7.3 \mathrm{~Hz}, \mathrm{CH}_{2}\right), 3.74(2 \mathrm{H}, \mathrm{d}, J=15.8 \mathrm{~Hz}, \mathrm{CH}$ thiazolidinone), $3.76\left(3 \mathrm{H}, \mathrm{s} \mathrm{OCH}_{3}\right), 6.08(1 \mathrm{H}, \mathrm{s}$ $\mathrm{CH}$ thiazolidinone), 6.95-7.30 (4H, m, Ar-H); ${ }^{13} \mathrm{C}$ NMR $\delta$ (ppm) $12.95\left(\mathrm{CH}_{3}\right.$ acyclic), 28.73 $\left(\mathrm{CH}_{2}\right.$ acyclic), 56.01 $\left(\mathrm{OCH}_{3}\right), 114.59-160.4$ (C of 
aromatic ring), 161.3, $156.8\left(\mathrm{C}_{2}, \mathrm{C}_{5}\right.$ of thiadiazole ring), $170.9(\mathrm{C}=\mathrm{O}$ Cyclic thiazolidinone), 34.3 $\left(\mathrm{CH}_{2}\right.$ thiazolidinone $), \quad 69.6 \quad(\mathrm{CH} \quad$ cyclic thiazolidinone); Anal. Calcd. For: $\mathrm{C}_{14} \mathrm{H}_{15} \mathrm{~N}_{4} \mathrm{O}_{2} \mathrm{~S}_{2}$ : C, 52.32, H, 4.70, N, $13.07 \%$; found $\mathrm{C}, 52.21, \mathrm{H}$, 4.40, N, $13.02 \%$; Mass $321\left(\mathrm{M}^{+}\right)$.

Synthesis of 5-benzylidene-3-(5-ethyl-[1,3,4] thiadiazol-2-yl)-2-phenyl-thiazolidin-4-ones

(3a): The compound-2 (0.002 mole,) and benzaldehyde $(0.002$ mole $)$ in methanol $(25 \mathrm{~mL})$ in the presence of sodium ethoxide undergo Knovenagel condensation. The reaction mixture was first continuously stirred on magnetic stirrer for about 2-3 hrs, then it was kept on steam bath for reflux for about $10 \mathrm{hr}$. After the completion of the reaction the excess of methanol was removed and the product thus obtained was washed with water and purified over a silica gel column and recrystallized from ethanol to yield compound 3; yellow crystals, m.p.-189-191 ${ }^{\circ} \mathrm{C}$, Yield $71 \%$ IR: 1453( $\left.v_{\mathrm{C}-\mathrm{C}}\right), 1545 \quad\left(v_{\mathrm{C}=\mathrm{C}}\right)$, $2949\left(v_{\mathrm{C}-\mathrm{H}}\right), 1470\left(v_{\mathrm{C}-\mathrm{N}}\right), 1310\left(v_{\mathrm{N}-\mathrm{N}}\right), 1207\left(v_{\mathrm{C}=\mathrm{N}}\right)$ $737\left(v_{\mathrm{C}-\mathrm{S}}\right), 1710\left(v_{\mathrm{C}=0}\right) ;{ }^{1} \mathrm{H}$ NMR: $\delta(\mathrm{ppm}) 1.09$ $\left(3 \mathrm{H}, \mathrm{t}, J=7.3 \mathrm{~Hz}, \mathrm{CH}_{3}\right), 2.79(2 \mathrm{H}, \mathrm{q}, J=7.3 \mathrm{~Hz}$, $\left.\mathrm{CH}_{2}\right), 6.40(1 \mathrm{H}, \mathrm{s}, \mathrm{CH}$ cyclic thiazolidinone), 7.21$7.65(10 \mathrm{H}, \mathrm{m}, \mathrm{Ar}-\mathrm{H}), 7.70$ (1H, s, CH acyclic); ${ }^{13} \mathrm{C}$ NMR: $\delta$ (ppm) $12.85\left(\mathrm{CH}_{3}\right.$ acyclic), $28.53\left(\mathrm{CH}_{2}\right.$ acyclic), 128.2-139.30 (C of aromatic ring), 161.3, $156.8\left(\mathrm{C}_{2}, \mathrm{C}_{5}\right.$ of thiadiazole ring $), 166.2(\mathrm{C}=\mathrm{O}$ Cyclic thiazolidinone); $69.5(\mathrm{CH} \quad$ cyclic thiazolidinone), 126.5 ( $\mathrm{CH}$ acyclic); Anal. Calcd. for: $\mathrm{C}_{20} \mathrm{H}_{17} \mathrm{~N}_{3} \mathrm{OS}_{2}$ : C, 63.30, H, 4. 52, N, 11.07, O, 4.22, S, $16.90 \%$; found C, 63.21, H, 4.34, N, 11.01 $\%$; Mass $379\left(\mathrm{M}^{+}\right)$.

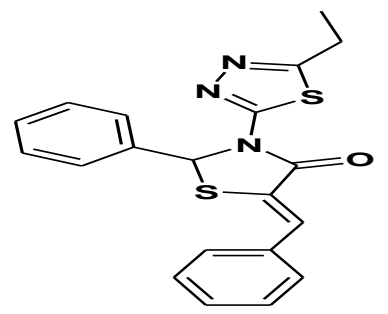

Compound-3a
The compounds $\mathbf{3 b - 3 m}$ were synthesized by the similar method as reported for $3 \mathrm{a}$.

5-(2-chloro-benzylidene)-2-(2-chloro-phenyl)-3(5-ethyl-[1,3,4]thiadiazole-2-yl)-thiazolidin-4one (3b) : m.p.169-171 ${ }^{\circ} \mathrm{C}$, yield $67 \%$ IR : $1451\left(v_{\mathrm{C}-\mathrm{C}}\right), 1555\left(v_{\mathrm{C}=\mathrm{C}}\right), 2949\left(v_{\mathrm{C}-\mathrm{H}}\right), 745\left(v_{\mathrm{C}-\mathrm{Cl}}\right)$, $1310\left(v_{\mathrm{N}-\mathrm{N}}\right), 1207\left(v_{\mathrm{C}=\mathrm{N}}\right) 737\left(v_{\mathrm{C}-\mathrm{S}}\right), 1714\left(v_{\mathrm{C}=\mathrm{O}}\right)$; ${ }^{1} \mathrm{H}$ NMR: $\delta$ (ppm) $1.18\left(3 \mathrm{H}, \mathrm{t}, J=7.3 \mathrm{~Hz}, \mathrm{CH}_{3}\right)$, $2.84\left(2 \mathrm{H}, \mathrm{q}, J=7.3 \mathrm{~Hz}, \mathrm{CH}_{2}\right), 6.39(1 \mathrm{H}, \mathrm{s}, \mathrm{CH}$ thiazolidinone), 7.21-7.80 (8H,m, Ar-H), 7.75 (1H, s, CH acyclic); ${ }^{13} \mathrm{C}$ NMR $\delta$ (ppm) 13.65 $\left(\mathrm{CH}_{3}\right.$ acyclic $), \quad 28.83\left(\mathrm{CH}_{2}\right.$ acyclic $), \quad 127.1-$ 134.3(C of aromatic ring), 160.4, $155.9\left(\mathrm{C}_{2}, \mathrm{C}_{5}\right.$ of thiadiazole ring $), \quad 165.2(\mathrm{C}=\mathrm{O} \quad$ cyclic thiazolidinone), 69.9 ( $\mathrm{CH}$ cyclic thiazolidinone), 125.9 ( $\mathrm{CH}$ acyclic); Anal. calcd. for: $\mathrm{C}_{20} \mathrm{H}_{15} \mathrm{Cl}_{2} \mathrm{~N}_{3} \mathrm{OS}_{2}$ : C, 53.57, H,3. 37, N, $9.37 \%$; found $\mathrm{C}, 53.31, \mathrm{H}, 3.14, \mathrm{~N}, 9.22 \%$; Mass 447 $\left(\mathrm{M}^{+}\right)$.

5-(3-chloro-benzylidene)-2-(3-chloro-phenyl)-3(5-ethyl-[1,3,4]thiadiazole-2-yl)-thiazolidin-4one (3c) : m.p.172-173 ${ }^{0} \mathrm{C}$, yield $65 \%$, IR $: 1451\left(v_{\mathrm{C}-\mathrm{C}}\right), 1551\left(v_{\mathrm{C}=\mathrm{C}}\right), 2959\left(v_{\mathrm{C}-\mathrm{H}}\right), 735\left(v_{\mathrm{C}-\mathrm{Cl}}\right)$, $1312\left(v_{\mathrm{N}-\mathrm{N}}\right), 1209\left(v_{\mathrm{C}=\mathrm{N}}\right)$ 736 $\left(v_{\mathrm{C}-\mathrm{S}}\right), 1720\left(v_{\mathrm{C}=\mathrm{O}}\right)$; ${ }^{1} \mathrm{H}$ NMR: $\delta(\mathrm{ppm}) 1.06\left(3 \mathrm{H}, \mathrm{t}, J=7.3 \mathrm{~Hz}, \mathrm{CH}_{3}\right)$, $2.88\left(2 \mathrm{H}, \mathrm{q}, J=7.3 \mathrm{~Hz}, \mathrm{CH}_{2}\right), 6.40(1 \mathrm{H}, \mathrm{s}, \mathrm{CH}$ thiazolidin-4-one), 7.22-7.89 (8H, m Ar-H), 7.84 $\left(1 \mathrm{H}, \quad \mathrm{s}, \quad \mathrm{CH}\right.$ acyclic); ${ }^{13} \mathrm{C}$ NMR: $\delta$ (ppm) 12.98 $\left(\mathrm{CH}_{3}\right.$ acyclic $), 28.23\left(\mathrm{CH}_{2}\right.$ acyclic $), 127.8-$ 132.2 ( $\mathrm{C}$ of aromatic ring), 161.4, $156.9\left(\mathrm{C}_{2}, \mathrm{C}_{5}\right.$ of thiadiazole) $167.2 \mathrm{C}=\mathrm{O}$ cyclic thiazolidinone, 68.5( $\mathrm{CH}$ cyclic thiazolidinone), 126.5 ( $\mathrm{CH}$ acyclic); Anal. Calcd. For: $\mathrm{C}_{20} \mathrm{H}_{15} \mathrm{Cl}_{2} \mathrm{~N}_{3} \mathrm{OS}_{2}$ : C, 53.57, H, 3. 37, N, $9.37 \%$; found C, 53.21, H, 3.21, N, $9.12 \%$, Mass 447( $\left.\mathrm{M}^{+}\right)$.

5-(4-chloro-benzylidene)-2-(4-chloro-phenyl)-3(5-ethyl-[1,3,4]thiadiazole-2-yl)-thiazolidin-4one (3d): m.p.170-172 ${ }^{\circ} \mathrm{C}$, yield $66 \%$ IR: $1453(v$ C-C $), 1554\left(v_{\mathrm{C}=\mathrm{C}}\right), 2979\left(v_{\mathrm{C}-\mathrm{H}}\right), \quad 741\left(v_{\mathrm{C}-\mathrm{Cl}}\right), 1312$ $\left(v_{\mathrm{N}-\mathrm{N}}\right), 1209\left(v_{\mathrm{C}=\mathrm{N}}\right), 734\left(\mathrm{~V}_{\mathrm{C}-\mathrm{S}}\right), 1713\left(\mathrm{~V}_{\mathrm{C}=\mathrm{O}}\right),{ }^{1} \mathrm{H}$ NMR: $\delta(\mathrm{ppm}) 1.12\left(3 \mathrm{H}, \mathrm{t}, J=7.3 \mathrm{~Hz}, \mathrm{CH}_{3}\right), 2.82$ 
$\left(2 \mathrm{H}, \mathrm{q}, J=7.3 \mathrm{~Hz}, \mathrm{CH}_{2}\right), \quad 6.41(1 \mathrm{H}, \mathrm{s}, \mathrm{CH}$ thiazolidinone ), $7.80(1 \mathrm{H}, \mathrm{s}, \mathrm{CH}$ acyclic), 7.497.75 (8H, m, Ar-H); ${ }^{13} \mathrm{C}$ NMR : $\delta$ (ppm) 13.25 $\left(\mathrm{CH}_{3}\right.$ acyclic $), 28.03\left(\mathrm{CH}_{2}\right.$ acyclic $), 129.5-139.3$ (C of aromatic ring), 160.7, 156.2 $\left(\mathrm{C}_{2}, \mathrm{C}_{5}\right.$ of thiadiazole ring $), \quad 166.5 \quad(\mathrm{C}=\mathrm{O} \quad$ cyclic thiazolidinone), $\quad 67.5(\mathrm{CH} \quad$ cyclic thiazolidinone), 126.4 (CH acyclic); Anal. Calcd. for: $\mathrm{C}_{20} \mathrm{H}_{15} \mathrm{Cl}_{2} \mathrm{~N}_{3} \mathrm{OS}_{2}$ : C, 53.57, H, 3. 37, N, $09.37 \%$; found $\mathrm{C}, 53.31, \mathrm{H}, 3.27, \mathrm{~N}, 9.20 \%$ ;Mass $447\left(\mathrm{M}^{+}\right)$.

5-(2-bromo-benzylidene)-2-(2-bromo-phenyl)-3(5-ethyl-[1,3,4]thiadiazole-2-yl)-thiazolidin-4one (3e) : m.p. 180-181 ${ }^{0} \mathrm{C}$, yield $70 \%$ IR: $1448\left(\mathrm{~V}_{\mathrm{C}-\mathrm{C}}\right), 1554\left(\mathrm{~V}_{\mathrm{C}=\mathrm{C}}\right), 2952\left(\mathrm{~V}_{\mathrm{C}-\mathrm{H}}\right), 535\left(\mathrm{v}_{\mathrm{C}-\mathrm{Br}}\right)$, $1316\left(v_{\mathrm{N}-\mathrm{N}}\right), 1211\left(v_{\mathrm{C}=\mathrm{N}}\right)$ 734( $\left(v_{\mathrm{C}-\mathrm{S}}\right), 1711\left(v_{\mathrm{C}=\mathrm{O}}\right)$; ${ }^{1} \mathrm{H}$ NMR: $\delta$ (ppm) $1.16\left(3 \mathrm{H}, \mathrm{t}, J=7.3 \mathrm{~Hz}, \mathrm{CH}_{3}\right)$, $2.86\left(2 \mathrm{H}, \mathrm{q}, J=7.3 \mathrm{~Hz}, \mathrm{CH}_{2}\right), 6.41(1 \mathrm{H}, \mathrm{s}, \mathrm{CH}$ thiazolidinone), 7.03-7.78 (8H, m, Ar-H) 7.78 (1H, s, CH acyclic); ${ }^{13} \mathrm{C}$ NMR: $\delta$ (ppm) 13.75 ( $\mathrm{CH}_{3}$ acyclic), 29.33 ( $\mathrm{CH}_{2}$ acyclic), 120.6-132.5 (C of aromatic ring), 161.4, 158.1 $\left(\mathrm{C}_{2}, \mathrm{C}_{5}\right.$ of thiadiazole ring $), \quad 165.2 \quad(\mathrm{C}=\mathrm{O} \quad$ cyclic thiazolidinone), 69.2 ( $\mathrm{CH}$ cyclic thiazolidinone), 126.8 (CH acyclic); Anal. Calcd. for: $\mathrm{C}_{20} \mathrm{H}_{15} \mathrm{Br}_{2}$ $\mathrm{N}_{3} \mathrm{OS}_{2}$ : C, 44.71, H, 2.81, N,7.82\%; found C, 44.56, H, 2.57, N, 7.66\%, Mass $537\left(\mathrm{M}^{+}\right)$.

5-(3-bromo-benzylidene)-2-(3-bromo-phenyl)-3(5-ethyl-[1,3,4]thiadiazole-2-yl)-thiazolidin-4one (3f): m.p.182-184 ${ }^{0} \mathrm{C}$, yield $72 \%$, IR:1445( $\left.v_{\mathrm{C}-\mathrm{C}}\right), 1552\left(v_{\mathrm{C}=\mathrm{C}}\right), 2962\left(v_{\mathrm{C}-\mathrm{H}}\right), \quad 540\left(v_{\mathrm{C}-}\right.$ $\mathrm{Br}), \quad 1313\left(v_{\mathrm{N}-\mathrm{N}}\right), \quad 1216\left(v_{\mathrm{C}=\mathrm{N}}\right) 736\left(v_{\mathrm{C}-\mathrm{S}}\right), 1715$ $\left(v_{\mathrm{C}=\mathrm{O}}\right) ;{ }^{1} \mathrm{H}$ NMR: $\delta(\mathrm{ppm}) 1.08(3 \mathrm{H}, \mathrm{t}, J=7.3 \mathrm{~Hz}$, $\left.\mathrm{CH}_{3}\right), 2.89\left(2 \mathrm{H}, \mathrm{q}, J=7.3 \mathrm{~Hz}, \mathrm{CH}_{2}\right), 6.41(1 \mathrm{H}, \mathrm{s}$, $\mathrm{CH}$ thiazolidin-4-one), 7.03-7.82 (8H, m, Ar-H) $7.81\left(1 \mathrm{H}, \mathrm{s}, \mathrm{CH}\right.$ acyclic); ${ }^{13} \mathrm{C}$ NMR $\delta$ (ppm) 11.95( $\mathrm{CH}_{3}$ acyclic), $26.83\left(\mathrm{CH}_{2}\right.$ acyclic $), 118.2-$ 137.5(C of aromatic ring), 162.4, $158.6\left(\mathrm{C}_{2}, \mathrm{C}_{5}\right.$ of thiadiazole),166.3 ( $\mathrm{C}=\mathrm{O}$ cyclic thiazolidinone), 68.5 (CH cyclic thiazolidinone),126.7 (CH acyclic); Anal. Calcd. for: $\mathrm{C}_{20} \mathrm{H}_{15} \mathrm{Br}_{2} \mathrm{~N}_{3} \mathrm{OS}_{2}$ : C,
44.71, H, 2.81, N, $7.82 \%$; found C, 44.56, H, 2.49, N, $7.50 \%$, Mass $537\left(\mathrm{M}^{+}\right)$.

5-(4-bromo-benzylidene)-2-(4-bromo-phenyl)-3(5-ethyl-[1,3,4]thiadiazole-2-yl)-thiazolidin-4one (3g) :m.p.181-183 ${ }^{0} \mathrm{C}$, yield $71 \%$, IR :1439( $\left(v_{\mathrm{C}-\mathrm{C}}\right), 1548\left(v_{\mathrm{C}=\mathrm{C}}\right), 2952\left(v_{\mathrm{C}-\mathrm{H}}\right), 546\left(v_{\mathrm{C}-\mathrm{Br}}\right)$, $1316\left(v_{\mathrm{N}-\mathrm{N}}\right), 1226\left(v_{\mathrm{C}=\mathrm{N}}\right) 733\left(v_{\mathrm{C}-\mathrm{S}}\right), 1719\left(v_{\mathrm{C}=\mathrm{O}}\right)$, ${ }^{1} \mathrm{H}$ NMR: $\delta(\mathrm{ppm}) 1.07\left(3 \mathrm{H}, \mathrm{t}, J=7.3 \mathrm{~Hz}, \mathrm{CH}_{3}\right)$, $2.81\left(2 \mathrm{H}, \mathrm{q}, J=7.3 \mathrm{~Hz}, \mathrm{CH}_{2}\right), 6.40(1 \mathrm{H}, \mathrm{s}, \mathrm{CH}$ thiazolidin-4-one), 7.30-7.74 (8H, m, Ar-H), $7.65\left(1 \mathrm{H}, \mathrm{s}, \mathrm{CH}\right.$ acyclic); ${ }^{13} \mathrm{C} \mathrm{NMR} \delta(\mathrm{ppm})$ 11.91 $\left(\mathrm{CH}_{3}\right.$ acyclic), $26.89\left(\mathrm{CH}_{2}\right.$ acyclic $), 127.8-$ 139.3 ( $\mathrm{C}$ of aromatic ring), 162.4, $158.6\left(\mathrm{C}_{2}, \mathrm{C}_{5}\right.$ of thiadiazole ring $), \quad 166.2 \quad(\mathrm{C}=\mathrm{O} \quad$ cyclic thiazolidineone), $\quad 69.4 \quad(\mathrm{CH} \quad$ cyclic thiazolidinone), 126.6(CH acyclic); Anal. Calcd. For: $\mathrm{C}_{20} \mathrm{H}_{15} \mathrm{Br}_{2} \mathrm{~N}_{3} \mathrm{OS}_{2}$ : C, 44.71, H, 2.81, 74, N, $7.82 \%$; found $\mathrm{C}, 44.66, \mathrm{H}, 2.59, \mathrm{~N}, 7.60 \%$; Mass $537\left(\mathrm{M}^{+}\right)$.

5-(2-nitro-benzylidene)-2-(2-nitro-phenyl)-3(5-ethyl-[1,3,4]thiadiazole-2-yl)-thiazolidin-4one (3h) : m.p. $185-187^{\circ} \mathrm{C}$, yield $60 \%$, IR:1448 $\left(v_{\mathrm{C}-\mathrm{C}}\right), 1460\left(v_{\mathrm{C}=\mathrm{C}}\right), 1314\left(v_{\mathrm{N}-\mathrm{N}}\right), 1630\left(v_{\mathrm{C}=\mathrm{N}}\right), 720$ $\left(v_{\mathrm{C}-\mathrm{S}}\right), 1716\left(v_{\mathrm{C}=\mathrm{O}}\right) 1538\left(v_{\mathrm{C}-\mathrm{NO}_{2}}\right), 735\left(v_{\mathrm{C}-\mathrm{N}}\right)$, ${ }^{1} \mathrm{H}$ NMR: $\delta(\mathrm{ppm}) 1.13\left(3 \mathrm{H}, \mathrm{t}, J=7.3 \mathrm{~Hz}, \mathrm{CH}_{3}\right)$, $2.84\left(2 \mathrm{H}, \mathrm{q}, J=7.3 \mathrm{~Hz}, \mathrm{CH}_{2}\right), 6.44(1 \mathrm{H}, \mathrm{s}, \mathrm{CH}$ thiazolidinone), 7.53-8.20 (8H, m,) Ar- $\mathrm{H}, 8.50$ (1H, s, CH acyclic); ${ }^{13} \mathrm{C}$ NMR $\delta$ (ppm) 121.8148.4( $\mathrm{C}$ of aromatic ring), $12.77\left(\mathrm{CH}_{3}\right.$ acyclic), 27.91 $\left(\mathrm{CH}_{2}\right.$ acyclic $), \quad 158.6,162.4 \quad\left(\mathrm{C}_{2}, \mathrm{C}_{5} \quad\right.$ of thiadiazole ring $), \quad 169.2 \quad(\mathrm{C}=\mathrm{O} \quad$ cyclic thiazolidinone), 69.5 (CH cyclic thiazolidinone), 127.1(CH acyclic); Anal. Calcd. for: $\mathrm{C}_{20} \mathrm{H}_{15} \mathrm{~N}_{3} \mathrm{O}_{5} \mathrm{~S}_{2} \mathrm{C}$, 51.16, H, 3.22, N, 14.92, $\mathrm{O}, 17.04, \mathrm{~S}, 13.66 \%$; found $\mathrm{C}, 51.06, \mathrm{H}, 2.12, \mathrm{~N}$, $13.32 \%$, Mass $469\left(\mathrm{M}^{+}\right)$.

\section{5-(3-nitro-benzylidene)-2-(3-nitro-phenyl)-3-} (5-ethyl-[1,3,4]thiadiazole-2-yl)-thiazolidin-4one (3i): m.p. $183-185^{\circ} \mathrm{C}$, yield $64 \%$, IR-1443 $\left(v_{\mathrm{C}-\mathrm{C}}\right), 1459\left(v_{\mathrm{C}=\mathrm{C}}\right), 1311\left(v_{\mathrm{N}-\mathrm{N}}\right), 1629\left(v_{\mathrm{C}=\mathrm{N}}\right), 735$ $\left(v_{\mathrm{C}-\mathrm{S}}\right), 1715\left(v_{\mathrm{C}=\mathrm{O}}\right) 1532\left(v \mathrm{C}-\mathrm{NO}_{2}\right), 735\left(v_{\mathrm{C}-\mathrm{N}}\right) ;{ }^{1} \mathrm{H}$ 
NMR: $\delta(\mathrm{ppm}) 1.16\left(3 \mathrm{H}, \mathrm{t}, J=7.3 \mathrm{~Hz}, \mathrm{CH}_{3}\right)$, $2.86(2 \mathrm{H}, \mathrm{q}, J=7.3 \mathrm{~Hz} \mathrm{CH}), 6.46(1 \mathrm{H}, \mathrm{s}, \mathrm{CH}$ thiazolidinone), 7.45-8.66 (8H, m, Ar-H), 7.96 (1H, s CH acyclic); ${ }^{13} \mathrm{C}$ NMR: $\delta$ (ppm) 116.4140.5 (C of aromatic ring), $12.72\left(\mathrm{CH}_{3}\right.$ acyclic), $27.83\left(\mathrm{CH}_{2}\right.$ acyclic $), 169.2, \quad 159.5 \quad\left(\mathrm{C}_{2}, \mathrm{C}_{5}\right.$ of thiadiazole ring $), \quad 167.2 \quad(\mathrm{C}=\mathrm{O} \quad$ cyclic thiazolidinone), $68.4(\mathrm{CH}$ cyclic thiazolidinone), 126.8 ( $\mathrm{CH}$ acyclic); Anal. Calcd. for : $\mathrm{C}_{20} \mathrm{H}_{15} \mathrm{~N}_{3}$ $\mathrm{O}_{5} \mathrm{~S}_{2}$ : C, 51.16, H, 3.22, N, $14.92 \%$; found $\mathrm{C}$, 51.06, H, 3.11, N, $14.62 \%$; Mass $469\left(\mathrm{M}^{+}\right)$.

5-(4-nitro-benzylidene)-2-(4-nitro-phenyl)-3(5-ethyl-[1,3,4]thiadiazole-2-yl)-thiazolidin-4one(3j) : m.p.190-191 ${ }^{\circ} \mathrm{C}$, yield $61 \%$, IR: 1439 $\left(v_{\mathrm{C}-\mathrm{C}}\right), 1540\left(v_{\mathrm{C}=\mathrm{C}}\right), 1321\left(v_{\mathrm{N}-\mathrm{N}}\right), 1633\left(v_{\mathrm{C}=\mathrm{N}}\right), 735$ $\left(v_{\mathrm{C}-\mathrm{S}}\right), 1717\left(v_{\mathrm{C}=\mathrm{O}}\right), 735\left(v_{\mathrm{C}-\mathrm{N}}\right), 1529\left(v, \mathrm{C}-\mathrm{NO}_{2}\right)$; ${ }^{1} \mathrm{H}$ NMR: $\delta(\mathrm{ppm}) 1.17\left(3 \mathrm{H}, \mathrm{t}, J=7.3 \mathrm{~Hz}, \mathrm{CH}_{3}\right)$, $2.85\left(2 \mathrm{H}, \mathrm{q}, J=7.3 \mathrm{~Hz}, \mathrm{CH}_{2}\right), 6.45(1 \mathrm{H}, \mathrm{s}, \mathrm{CH}$ thiazolidinone), 7.44-8020 (8H, m, Ar-H), 8.26 (1H, s, CH acyclic); ${ }^{13} \mathrm{C}$ NMR $: \delta$ (ppm) 117.3140.5 (C of aromatic ring), $12.70\left(\mathrm{CH}_{3}\right.$ acyclic), 27.73 $\left(\mathrm{CH}_{2}\right.$ acyclic), 169.2, $159.5\left(\mathrm{C}_{2}, \mathrm{C}_{5}\right.$ of thiadiazole ring $), \quad 170.3 \quad(\mathrm{C}=\mathrm{O} \quad$ cyclic thiazolidinone), $67.8(\mathrm{CH}$ cyclic thiazolidinone), 126.5 ( $\mathrm{CH}$ acyclic); Anal. Calcd. For: $\mathrm{C}_{20} \mathrm{H}_{15} \mathrm{~N}_{3}$ $\mathrm{O}_{5} \mathrm{~S}_{2}: \mathrm{C}, 51.16, \mathrm{H}, 3.22, \mathrm{~N}, 14.92 \%$; found $\mathrm{C}$, 51.06, H, 3.11, N, $14.52 \%$; Mass $469\left(\mathrm{M}^{+}\right)$.

\section{5-(2-methoxy-benzylidene)-2-(2-methoxy-} phenyl)-3-(5-ethyl-[1,3,4]thiadiazole-2-yl)thiazolidin-4-one (3k): m.p. $133-135^{\circ} \mathrm{C}$, yield $76 \%$, IR:1439 ( $\left.v_{\mathrm{C}-\mathrm{C}}\right), 1540\left(v_{\mathrm{C}=\mathrm{C}}\right), 1319\left(v_{\mathrm{N}-\mathrm{N}}\right)$, $1718\left(v_{\mathrm{C}=\mathrm{O}}\right), 1633\left(v_{\mathrm{C}=\mathrm{N}}\right), 734\left(v_{\mathrm{C}-\mathrm{S}}\right), 2971,(v$, $\left.\mathrm{OCH}_{3}\right) ;{ }^{1} \mathrm{H}$ NMR: $\delta$ (ppm) $1.13(3 \mathrm{H}, \mathrm{t}, J=7.3$ $\left.\mathrm{Hz}, \mathrm{CH}_{3}\right), 2.80(2 \mathrm{H}, \mathrm{q}, J=7.3 \mathrm{~Hz}) \mathrm{CH}_{2}, 3.71-$ $3.86\left(6 \mathrm{H}, \quad \mathrm{s}, \quad \mathrm{OCH}_{3}\right), \quad 6.32(1 \mathrm{H}, \quad \mathrm{s}, \quad \mathrm{CH}$ thiazolidinone ), 6.74-7.78 (8H, m, Ar-H). 7.59 (1H, s, CH acyclic); ${ }^{13} \mathrm{C}$ NMR: $\delta$ (ppm) 12.71 ( $\mathrm{CH}_{3}$ acyclic), 26.83 $\left(\mathrm{CH}_{2}\right.$ acyclic $), 55.4\left(\mathrm{OCH}_{3}\right)$, 111.4-156.4 (C of aromatic ring), 168.3, 155.8 $\left(\mathrm{C}_{2}, \mathrm{C}_{5}\right.$ of thiadiazole ring $), 170.2(\mathrm{C}=\mathrm{O}$ cyclic thiazolidinone), 68.5 ( $\mathrm{CH}$ cyclic thiazolidinone), 127.3 ( $\mathrm{CH}$ acyclic); Anal. Calcd. for: $\mathrm{C}_{22} \mathrm{H}_{21} \mathrm{~N}_{3}$
$\mathrm{O}_{3} \mathrm{~S}_{2}: \mathrm{C}, 60.11, \mathrm{H}, 4.28, \mathrm{~N}, 9.56 \%$; found $\mathrm{C}$, 60.06, H, 4.19.01, N, 9.26.12\%; Mass 438 $\left(\mathrm{M}^{+}\right)$.

\section{5-(3-methoxy-benzylidene)-2-(3-methoxy-}

\section{phenyl)-3-(5-ethyl-[1,3,4]thiadiazole-2-yl)-}

thiazolidin-4-one (3l) -m.p. $133-134{ }^{0} \mathrm{C}$, yield $75 \%$, IR: $1441\left(v_{\mathrm{C}-\mathrm{C}}\right), 1542\left(v_{\mathrm{C}=\mathrm{C}}\right), 1317\left(v_{\mathrm{N}-\mathrm{N}}\right)$, $1712\left(v_{\mathrm{C}=\mathrm{O}}\right), 1636\left(v_{\mathrm{C}=\mathrm{N}}\right), 736\left(v_{\mathrm{C}-\mathrm{S}}\right), 2970(v$ $\left.\mathrm{OCH}_{3}\right) ;{ }^{1} \mathrm{H}$ NMR: $\delta(\mathrm{ppm}) 1.14(3 \mathrm{H}, \mathrm{t}, J=7.3$ $\left.\mathrm{Hz}, \mathrm{CH}_{3}\right), 2.81\left(2 \mathrm{H}, \mathrm{q}, J=7.3 \mathrm{~Hz}, \mathrm{CH}_{2}\right), 3.73-$ $3.75\left(6 \mathrm{H}, \mathrm{s}, \mathrm{OCH}_{3}\right), 6.26(1 \mathrm{H}, \mathrm{s}, \mathrm{CH}$ thiazolidin4-one), 6.88-7.50 (8H,m, Ar-H), 7.81 (1H, s, CH acyclic); ${ }^{13} \mathrm{C}$ NMR $\delta$ (ppm) 111.3-157.7(C of aromatic ring $), 13.15\left(\mathrm{CH}_{3}\right.$ acyclic $), 26.32\left(\mathrm{CH}_{2}\right.$ acyclic), 155.8, 168.3, $\left(\mathrm{C}_{2}, \mathrm{C}_{5}\right.$ of thiadiazole ring $), \quad 167.6 \quad(\mathrm{C}=\mathrm{O}$ cyclic thiazolidineone $)$, 68.7( $\mathrm{CH}$ cyclic thiazolidinone), 127.3(CH acyclic); Anal. Calcd. For: $\mathrm{C}_{22} \mathrm{H}_{21} \mathrm{~N}_{3} \mathrm{O}_{3} \mathrm{~S}_{2}$ : C, 60.11, H,4.28, N, $9.56 \%$; found C, 60.06, H, 4.15, N, $9.36 \%$; Mass $439\left(\mathrm{M}^{+}\right)$.

\section{5-(4-methoxy-benzylidene)-2-(4-methoxy-} phenyl)-3-(5-ethyl-[1,3,4]thiadiazole-2-yl)thiazolidin-4-one (3m) :m.p.137-139 ${ }^{0} \mathrm{C}$, yield $75 \%$, IR: $1443\left(v_{\mathrm{C}-\mathrm{C}}\right), 1540\left(v_{\mathrm{C}=\mathrm{C}}\right), 1319\left(v_{\mathrm{N}-\mathrm{N}}\right)$, $1715\left(v_{\mathrm{C}=\mathrm{O}}\right), 1638\left(v_{\mathrm{C}=\mathrm{N}}\right), 734\left(v_{\mathrm{C}-\mathrm{S}}\right), 2969(v$ $\left.\mathrm{OCH}_{3}\right) ;{ }^{1} \mathrm{H}$ NMR: $\delta$ (ppm) $1.12(3 \mathrm{H}, \mathrm{t}, J=7.3$ $\left.\mathrm{Hz}, \mathrm{CH}_{3}\right), 2.78\left(2 \mathrm{H}, \mathrm{q}, J=7.3 \mathrm{~Hz}, \mathrm{CH}_{2}\right), 3.76-$ $3.79\left(6 \mathrm{H}, \quad \mathrm{s}, \mathrm{OCH}_{3}\right), \quad 6.38(1 \mathrm{H}, \quad \mathrm{s}, \mathrm{CH}$ thiazolidinone), 6.95-7.73 (8H, m, Ar-H). 7.65 (1H, s, CH cyclic); ${ }^{13} \mathrm{C}$ NMR: $\delta$ (ppm) 114.3160.3 (C of aromatic ring), $12.65\left(\mathrm{CH}_{3}\right.$ acyclic), $26.38\left(\mathrm{CH}_{2}\right.$ acyclic $), 167.6,156.9\left(\mathrm{C}_{2}, \mathrm{C}_{5}\right.$ of thiadiazole ring $), \quad 169.4 \quad(\mathrm{C}=\mathrm{O} \quad$ cyclic thiazolidineone), 67.5(CHcyclic thiazolidinone), 126.3( $\mathrm{CH}$ acyclic); Anal. Calcd. For: $\mathrm{C}_{22} \mathrm{H}_{21} \mathrm{~N}_{3}$ $\mathrm{O}_{3} \mathrm{~S}_{2}$ : C, 60.11, H,4.28, N, $9.56 \%$; found C, 60.00, H, 4.15, N, 9.44\%, Mass $439\left(\mathrm{M}^{+}\right)$.

\section{RESULTS AND DISCUSSION}

The reaction of 2-amino-5-ethyl-1,3,4-thiadiazole and benzaldehyde afforded compound 1a. The 
spectroscopic analyses of compound 1a showed absorption peaks for $\mathrm{N}-\mathrm{CH}$ for Schiff bases $\mathrm{N}=\mathrm{CH}$ are confirmed by IR, ${ }^{1} \mathrm{H}$ NMR and ${ }^{13} \mathrm{C}$ NMR spectra. $\mathbf{I a}-\mathbf{1 m}$ have been synthesized by taking various derivatives of benzaldehyde. In the IR spectra an absorption found in the range of $1665-1675 \mathrm{~cm}^{-1}$, and a strong signal in the range of $\delta 9.34-9.46$ and $\delta 158-161 \mathrm{ppm}$ in the afford compounds $\mathbf{3 a}-\mathbf{3} \boldsymbol{m}$. In the ${ }^{1} \mathrm{H}$ NMR spectra of the compounds disappearance of two methylene protons of $\mathbf{2 a - 2 m}$ and appearance of a new signal for $\mathrm{C}=\mathrm{CH}$ in the range of $\delta 7.39$ $8.50 \mathrm{ppm}$ in the ${ }^{1} \mathrm{H}$ NMR was observed. Two new signals for $\mathrm{C}=\mathrm{CH}$ appeared in the range of $\delta 125-130 \mathrm{ppm}$ and $\mathrm{C}-\mathrm{H}$ appeared in the range of $\delta 67-70 \mathrm{ppm}$ in the ${ }^{13} \mathrm{C}$ NMR spectra of $3 \boldsymbol{a}-$ $3 \mathbf{m}$. These facts clearly confirmed the formation

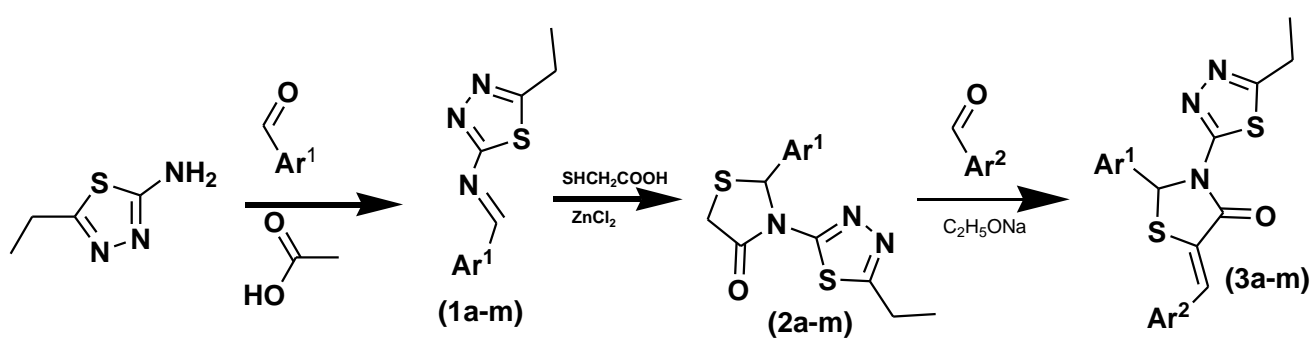

$A r^{1}, A r^{2}=$ Various Substituted Aryl Groups

${ }^{1} \mathrm{H}$ NMR and ${ }^{13} \mathrm{C}$ NMR spectra respectively supported the formation of compounds $\mathbf{1 a}-\mathbf{1 m}$. The fact is also supported by the disappearance of the signal of $\mathrm{NH}_{2}$ in the ${ }^{1} \mathrm{H}$ NMR spectra.

The compounds $\mathbf{1 a}-\mathbf{1 m}$ on reaction with equimolar amount of thioglycolic acid in the presence of $\mathrm{ZnCl}_{2}$ in trace amount gives the cycloaddition reaction and produced a five membered thiazolidinone ring, $2 a-2 m$. The compound $\mathbf{2 a - 2 m}$ showed a characteristic absorption of the cyclic carbonyl group in the range of $1710-1721 \mathrm{~cm}^{-1}$ in the IR spectra. The ${ }^{1} \mathrm{H}$ NMR spectra clearly indicate the presence of the active methylene group in the thiazolidine ring in the range of $\delta 3.61-3.96 \mathrm{ppm}$. The ${ }^{13} \mathrm{C}$ NMR spectra of compounds $\mathbf{2 a}-\mathbf{2 m}$ also supported the presence of cyclic carbonyl group where a ${ }^{13} C$ signal appeared in the range of $\delta$ 165.4-175.2 ppm. These are supported by two evidences that are; (a) disappearance of $\mathrm{N}=\mathrm{CH}$ proton and (b) appearance of $\mathrm{N}-\mathrm{CH}$ proton in the range of $\delta$ 6.08-6.37 (acyclic $\mathrm{CH}$ ) ppm. in the ${ }^{1} \mathrm{H}$ NMR spectra of compounds $\mathbf{2 a}-\mathbf{2} \boldsymbol{m}$. Compounds $\mathbf{2 a - 2 m}$ underwent the Knoevenagel condensation reaction with substituted benzaldehydes in the presence of $\mathrm{C}_{2} \mathrm{H}_{5} \mathrm{ONa}$ to of compounds $2 a-2 m$ and $3 a-3 m$.

\section{Antimicrobial activities}

The synthesized compounds $\mathbf{2 a - 2 m}$ and $\mathbf{3 a}-\mathbf{3 m}$ were evaluated in vitro for antibacterial activity by using filter paper disc diffusion method against different strains of bacteria viz. $B$. substilis, E. Coli and S. tyhpi. All the final products along with standard antibacterial streptomycin were used at 50 and $100 \mathrm{ppm}$ concentrations. Antifungal activity against A.niger, A. Flavus and F. oxisporium at 50 and $100 \mathrm{mg} / \mathrm{mL}$ concentrations was also determined by filter paper disc technique. The minimum inhibitory concentration (MIC) values of the synthesized compounds were determined. Standard antibacterial Streptomycin and antifungal griseofulvin were also tested under the similar conditions for comparison (Tables: 1, 2 and 3).

Nitro group containing compounds showed higher activity in the order $(\mathbf{3} \boldsymbol{i}>\mathbf{3} \boldsymbol{j}>\mathbf{3} \boldsymbol{h})$ than chloro $(\mathbf{3} \boldsymbol{c}>\mathbf{3} \boldsymbol{b}>\mathbf{3 d})$, or bromo group containing compounds $(\mathbf{3} f>3 g>3 e)$. Similar order of activity for compounds $\mathbf{2 a}-\mathbf{2 m}$ was observed. The chloro and bromo derivatives also have 
higher activity than other compounds in the series. On the basis of Structural Activity Relationship (SAR),it can be concluded that the activity of compounds depends on electron withdrawing nature of the substituted groups, $\mathrm{NO}_{2}>-\mathrm{Cl}>-\mathrm{Br}>-\mathrm{OCH}_{3}$.

Table 1. Antibacterial Activity (Inhibition zone diameter in $\mathbf{m m}$ ) of Compounds 2a-2m and 3a-3m

\begin{tabular}{|c|c|c|c|c|c|c|}
\hline \multirow[t]{2}{*}{ Comp. } & \multicolumn{2}{|c|}{ E. coli } & \multicolumn{2}{|c|}{ B. subtilis } & \multicolumn{2}{|c|}{ S. Typhi } \\
\hline & $50 \mathrm{mg} / \mathrm{mL}$ & $100 \mathrm{mg} / \mathrm{mL}$ & $50 \mathrm{mg} / \mathrm{mL}$ & $100 \mathrm{mg} / \mathrm{mL}$ & $50 \mathrm{mg} / \mathrm{mL}$ & $100 \mathrm{mg} / \mathrm{mL}$ \\
\hline $2 a$ & 6 & 9 & 4 & 5.3 & 4 & 7 \\
\hline $2 b$ & 15.9 & 16 & 14.6 & 16.9 & 14 & 17 \\
\hline $2 \mathrm{c}$ & 16.7 & 18.4 & 14 & 16.2 & 15.3 & 18 \\
\hline $2 d$ & 15.4 & 19 & 15 & 17.9 & 16 & 19.4 \\
\hline $2 \mathrm{e}$ & 13 & 14.2 & 12.2 & 15.8 & 11.9 & 11.5 \\
\hline $2 f$ & 12.8 & 13.6 & 11.7 & 14.9 & 12 & 16 \\
\hline $2 \mathrm{~g}$ & 13.5 & 14.8 & 13 & 16.2 & 12.6 & 16.5 \\
\hline $2 \mathrm{~h}$ & 18 & 23.5 & 18.3 & 22.5 & 18.9 & 24.7 \\
\hline $2 \mathrm{i}$ & 19 & 24.8 & 19 & 24 & 18.7 & 24.8 \\
\hline $2 \mathrm{j}$ & 17.5 & 24.5 & 17.5 & 23.5 & 17.5 & 23.8 \\
\hline $2 \mathrm{k}$ & 12.5 & 14 & 10 & 12.5 & 10.2 & 13.7 \\
\hline 21 & 11.8 & 13.2 & 10.6 & 13.2 & 11 & 14.6 \\
\hline $2 \mathrm{~m}$ & 12.6 & 13.9 & 11.3 & 13.8 & 11.5 & 14.9 \\
\hline $3 a$ & 8.9 & 11.2 & 7 & 9.8 & 7.5 & 10.6 \\
\hline $3 b$ & 15.2 & 18.3 & 15.4 & 18.2 & 14.5 & 19.3 \\
\hline $3 c$ & 16.5 & 19.2 & 16 & 19.8 & 15 & 19 \\
\hline $3 d$ & 14.9 & 18 & 14.8 & 17.6 & 15.2 & 29.2 \\
\hline $3 e$ & 12.6 & 14.5 & 12.8 & 16.9 & 13 & 16 \\
\hline $3 \mathrm{f}$ & 13 & 15.9 & 13 & 17 & 13.9 & 17 \\
\hline $3 g$ & 12.5 & 14.8 & 12.5 & 15.5 & 13.5 & 21.8 \\
\hline $3 \mathrm{~h}$ & 21.7 & 26.9 & 20.6 & 25.7 & 21.2 & 25 \\
\hline $3 \mathrm{i}$ & 22.3 & 26 & 21.5 & 26.3 & 22.5 & 26 \\
\hline $3 \mathrm{j}$ & 22 & 25.5 & 22.2 & 27 & 22 & 26.5 \\
\hline $3 \mathrm{k}$ & 12 & 16 & 12.3 & 15 & 12 & 16 \\
\hline 31 & 11 & 15 & 9.9 & 12.4 & 10.1 & 13.8 \\
\hline $3 \mathrm{~m}$ & 12.4 & 15.9 & 11.4 & 13.7 & 11.7 & 14.8 \\
\hline $\mathrm{SM}^{\mathrm{a}}$ & 26.3 & 29.5 & 23 & 27 & 25 & 28 \\
\hline
\end{tabular}


Table 2. Antifungal Activity (Inhibition Zone diameter in $\mathrm{mm}$ ) of the Compounds 2a-2m and 3a-3m

\begin{tabular}{|c|c|c|c|c|c|c|}
\hline \multirow[t]{2}{*}{ Comp. } & \multicolumn{2}{|c|}{ A. flavus } & \multicolumn{2}{|c|}{ A.niger } & \multicolumn{2}{|c|}{ F. oxisporium } \\
\hline & $50 \mathrm{mg} / \mathrm{mL}$ & $100 \mathrm{mg} / \mathrm{mL}$ & $50 \mathrm{mg} / \mathrm{mL}$ & $100 \mathrm{mg} / \mathrm{mL}$ & $50 \mathrm{mg} / \mathrm{mL}$ & $100 \mathrm{mg} / \mathrm{mL}$ \\
\hline $2 \mathrm{a}$ & 6.0 & 9.0 & 6.5 & 8.7 & 8.6 & 10.2 \\
\hline $2 b$ & 14.7 & 17.0 & 14.5 & 17.5 & 15.5 & 18.4 \\
\hline $2 \mathrm{c}$ & 14.9 & 17.5 & 15.9 & 18.6 & 14.6 & 17.7 \\
\hline $2 \mathrm{~d}$ & 14.2 & 20.5 & 16.0 & 18.6 & 16.2 & 19.5 \\
\hline $2 e$ & 12.5 & 16.8 & 10.0 & 13.3 & 11.0 & 13.9 \\
\hline $2 \mathrm{f}$ & 11.6 & 14.5 & 10.5 & 14.5 & 12.8 & 16.6 \\
\hline $2 \mathrm{~g}$ & 13.0 & 17.9 & 11.9 & 14.5 & 13.0 & 16.2 \\
\hline $2 \mathrm{~h}$ & 17.4 & 24.0 & 18.5 & 23.5 & 18.5 & 24.0 \\
\hline $2 \mathrm{i}$ & 17.5 & 24.5 & 19.0 & 25.0 & 19.5 & 24.5 \\
\hline $2 \mathrm{j}$ & 17.2 & 23.0 & 18.0 & 24.0 & 18.0 & 23.5 \\
\hline $2 \mathrm{k}$ & 10.9 & 14.1 & 10.0 & 13.5 & 12.0 & 15.2 \\
\hline 21 & 8.8 & 11.5 & 7.0 & 10.0 & 10.3 & 13.4 \\
\hline $2 \mathrm{~m}$ & 11.2 & 13.6 & 8.6 & 11.5 & 10.4 & 13.9 \\
\hline $3 a$ & 9.0 & 11.5 & 7.0 & 9.5 & 8.0 & 11.0 \\
\hline $3 b$ & 16.0 & 20.0 & 14.8 & 19.5 & 17.0 & 21.5 \\
\hline $3 c$ & 18.0 & 23.4 & 16.4 & 22.0 & 16.5 & 23.2 \\
\hline $3 d$ & 17.5 & 22.5 & 15.2 & 21.4 & 17.5 & 21.8 \\
\hline $3 e$ & 12.8 & 18.9 & 13.5 & 16.8 & 11.5 & 14.6 \\
\hline $3 \mathrm{f}$ & 13.0 & 16.8 & 12.6 & 15.0 & 13.8 & 16.7 \\
\hline $3 g$ & 14.9 & 18.2 & 11.4 & 13.6 & 12.0 & 15.0 \\
\hline $3 \mathrm{~h}$ & 21.7 & 27.0 & 21.0 & 26.5 & 21.0 & 27.0 \\
\hline $3 \mathrm{i}$ & 22.5 & 26.8 & 21.5 & 26.0 & 20.5 & 26.0 \\
\hline $3 j$ & 20.0 & 26.0 & 20.5 & 26.8 & 22.0 & 26.5 \\
\hline $3 \mathrm{k}$ & 11.0 & 14.0 & 10.0 & 13.3 & 10.3 & 14.5 \\
\hline 31 & 10.5 & 14.4 & 11.4 & 14.6 & 11.0 & 15.5 \\
\hline $3 \mathrm{~m}$ & 11.5 & 15.4 & 11.4 & 14.4 & 10.5 & 15.4 \\
\hline $\mathrm{GF}^{\mathrm{b}}$ & 27.0 & 30.0 & 25.0 & 28.0 & 28.0 & 32.0 \\
\hline
\end{tabular}

\section{CONCLUSION}

A new series of compounds $\mathbf{2 a}-2 \mathbf{m}$ and $\mathbf{3 a - 3} \mathbf{m}$ were synthesized and the synthesized compounds were screened for their biological study. The investigation of antimicrobial (antibacterial and antifungal) activities data revealed that the compounds follow the order ( $2 \mathbf{i}$ $>2 \mathrm{~h}>2 \mathrm{j}>2 \mathrm{c}>2 \mathrm{~b}>2 \mathrm{~d}>2 \mathrm{~g})$ and $(3 \mathrm{i}>3 \mathrm{j}>3 \mathrm{~h}>$ 3c $>3 \mathbf{d}>3 \mathbf{b}$ and 3f) and displayed excellent activity; the compounds follow the order $(\mathbf{2 e}>\mathbf{2 f}$ 
Table 3. Minimal inhibitory concentrations (MIC) in $\mathrm{mg} / \mathrm{mL}$ of synthesized compounds against bacterial and fungal strains

\begin{tabular}{|c|c|c|c|c|c|c|}
\hline Compound & E. coli & B. subtilis & S. typhi & A. flavus & A.niger & F. oxisporium \\
\hline $2 a$ & 32 & 44 & 37 & 33 & 39 & 30 \\
\hline $2 b$ & 16 & 21 & 19 & 19 & 23 & 14 \\
\hline $2 c$ & 17 & 18 & 17 & 17 & 21 & 14 \\
\hline $2 \mathrm{~d}$ & 18 & 19 & 18 & 19 & 22 & 15 \\
\hline $2 \mathrm{e}$ & 21 & 23 & 21 & 22 & 27 & 16 \\
\hline $2 \mathrm{f}$ & 20 & 23 & 20 & 23 & 25 & 16 \\
\hline $2 \mathrm{~g}$ & 22 & 24 & 21 & 25 & 24 & 16 \\
\hline $2 \mathrm{~h}$ & 14 & 16 & 13 & 15 & 17 & 11 \\
\hline $2 \mathrm{i}$ & 14 & 15 & 13 & 14 & 16 & 11 \\
\hline $2 \mathrm{j}$ & 15 & 16 & 14 & 12 & 15 & 10 \\
\hline $2 \mathrm{k}$ & 27 & 37 & 31 & 22 & 35 & 20 \\
\hline 21 & 25 & 38 & 26 & 21 & 32 & 18 \\
\hline $2 \mathrm{~m}$ & 26 & 27 & 30 & 23 & 31 & 18 \\
\hline $3 a$ & 30 & 45 & 38 & 33 & 38 & 31 \\
\hline $3 b$ & 17 & 19 & 18 & 17 & 18 & 11 \\
\hline $3 c$ & 18 & 20 & 18 & 15 & 19 & 10 \\
\hline $3 d$ & 19 & 21 & 21 & 19 & 19 & 11 \\
\hline $3 e$ & 20 & 22 & 20 & 20 & 27 & 13 \\
\hline $3 \mathrm{f}$ & 21 & 21 & 21 & 18 & 26 & 14 \\
\hline $3 g$ & 21 & 20 & 21 & 19 & 28 & 13 \\
\hline $3 \mathrm{~h}$ & 12 & 15 & 13 & 12 & 13 & 09 \\
\hline $3 \mathrm{i}$ & 13 & 15 & 12 & 12 & 13 & 08 \\
\hline $3 j$ & 14 & 16 & 14 & 13 & 14 & 08 \\
\hline $3 \mathrm{k}$ & 26 & 36 & 30 & 22 & 34 & 19 \\
\hline 31 & 25 & 37 & 26 & 21 & 33 & 17 \\
\hline $3 \mathrm{~m}$ & 25 & 35 & 29 & 23 & 32 & 17 \\
\hline Streptomycin & 9 & 13 & 11 & --- & --- & ---- \\
\hline Griseofulvin & --- & --- & --- & 9 & 11 & 03 \\
\hline
\end{tabular}

$>\mathbf{2 m}>\mathbf{2 k})$ and $(\mathbf{3 e}>\mathbf{3 g}>\mathbf{3 m}>\mathbf{3 k})$ displayed moderate activity and the other compounds showed less activity compared with standard drugs.

\section{ACKNOWLEDGEMENT}

The authors are thankful to SAIF, Punjab University, Chandigarh (India) for providing spectral and analytical data of the compounds. 
The authors are also grateful to Mr. Vahid-UlHassan, and Head department of Zoology Dr. H.S. Gour University, Sagar (M.P.) for providing and help in carrying out the antimicrobial screening. The authors are also thankful to Head, Department of Chemistry, Dr. H. S. Gour, Central University Sagar (India) for giving the facilities to carry out the work. and UGC-New Delhi (India), for financial assistance as R.G.N.F. fellowship.

\section{REFERENCE}

Aamer, S., Naeem, A., Ulrich, F. 2007. Synthesis and antibacterial activity of some novel 2-arollimino-3 aryl-thizolidin-4-one. J. Braz. Chem. Soc. 18(3): 559-65

Chaudhary, M., Parmar, S., Chaudhary, S.K., Chaturvedi, A.K., Ramasastry, B.V. 1976. CNS depressant activity of pyrimidyl thiazolidinones. J. Pharm. Sci. 64: 443-448.

Dua, R., Shrivastava S., Sonwane, S.K., Srivastava, S.K. 2011. Pharmacological significance of synthetic heterocycles scaffold: A Review. Advances in Biological Research. 5(3): 120-144.

Eghbalian, A. E., Kiani, A. 2013.Synthesis and Evaluation of the Cytotoxicity of a Series of 1,3,4-Thiadiazole Based Compounds as Anticancer Agents. Iran J Basic Med Sci. 16(11): 1133-1138.

Fu, X.S., Li, Jing F., Wang, X., Li B, Zhao, J., Liu Y., Chen, B. 2016. Synthesis and Biological Evaluation of Novel 1,3,4thiadiazole Derivatives Incorporating Benzisoselenazolone Scaffold as Potential Antitumor Agents. Med Chem. 12(7): 631639.

Goel, B., Ram, T., Tyagi, R., Bansal, E., Kumar, A., Mukherjee, D., Sinha, J.N. 1999. 2Substituted-3-(4-bromo-2-carboxyphenyl)5-methyl-4-thiazolidinones as potential anti-inflammatory agents. Eur. J. Med. Chem. 34: 265-69.

Haider, S., Alam, M. S., Hamid, H. 2015. 1,3,4Thiadiazoles: A potent multi targeted pharmacological scaffold. Eur. J. Med. Chem. 92: 156-177.

Jain, A.K., Sharma, S., Vaidya, A., Veerasamy, R., Agrawal, R. K. 2013. 1,3,4-Thiadiazole and its Derivatives: A Review on Recent Progress in Biological Activities. Chem. Bio. Drug. Res. 181: 557-576.

Jubie, S., Gowramma, B., Nitin, K.M., Jawahar, N.R., Kalirajan, Gomathy, S. 2009. Synthesis and biological evaluation of some 3- (methoxy phenyl)- 2- aryl-thiazolidin-4one derivatives. Indian. J. Pharm. Sci. 1(1): 32-38.

Kandapalli, V.G., Shekar, C., Rao, V.S. 2010. Solvent free microwave accelerated synthesis of heterocyclic thiazolidine-4-ones as antimicrobial and antifungal agents. Bull Korean Chem. Soc. 35(5): 1219-22.

Li, Y., Geng J., Liu, Y., Yu S., Zhao, G. 2013. Thiadiazole A Promising Structure in Medicinal Chemistry. Chem. Med. Chem. 8: 27-41.

Mehlika, D.A., Ozgu, DCr., Umide, D.O., Zafer, A. K. 2016. Synthesis and Evaluation of New 1,3,4-Thiadiazole Derivatives as Antinociceptive Agents. Molecules. 21:1004-1014.

Murugesan, S., Srinivasan, S., Marimuthu, P. 2012. Synthesis, Characterization and Biological Evaluation of 4-Thiazolidinone Derivatives. J. Pharm. Bio. Sci. 16(13): 1-5.

Pattanayak, P., Sharma, R., Sahoo, P. K. 2009. Synthesis and evaluation of 2-amino-5sulfanyl-1,3,4-thiadiazoles as antidepressant, anxiolytic, and anticonvulsant agents. Med. Chem. Res. 18: 351-361. 
Parmeshwaran, M., Gopalkrishnan, S. 2009. Synthesis of coumarin heterocyclic derivatives withantioxidant activity and in vitro cytotoxic activity against tumour cells. Acta Pharm. 59: 159-170.

Rajak, H., Deshmukh, R., Aggarwal, N., Kashaw S., Kharya, M.D., Mishra, P. 2009. Synthesis of novel 2,5-disubstituted 1,3,4thiadiazoles for their potential anticonvulsant activity: Pharmacophoric model studies. Arch. Pharm. Chem. Life Sci. 342: 453-461.

Rawal, R. 2005. 2-(Aryl) -3-furan-2ylmethylthiazolidin-4-ones as selective HIV-RT inhibitors. Bioorg. Med. Chem. 13: 6771-6776.

Sharma, R., Samadhiya, P., Srivastava, S.D., Srivastava, S.K. 2012. Synthesis and biological activity of 4-thiazolidinone derivatives of phenothiazine. J. Serb. Chem. Soc. 77(1): 17-26.
Shkair, A.M., Shakya, A.K., R Raghavendra, N.M., and Naik, R. 2016. Molecular Modeling, Synthesis and Pharmacological Evaluation of 1,3,4-Thiadiazoles as Antiinflammatory and Analgesic Agents. Med. Chem. 12: 90-100.

War, J., Shrivastav, S. K., Shrivastava, S.D. 2017. Design ,Synthesis and DNA Binding Study of some novel morpholine linked thiazolidinone derivatives. Spectrochimica Acta Part A: Molecular and Biomolecular Spectroscopy. 173: 270-278.

Zhuo, C., Weiming, X., Keming, L., Song, Y., Huitao, F., Pinak, S.B., De-Yu H., Yuping, Z. 2010. Synthesis and Antiviral Activity of 5-(4-Chlorophenyl)-1,3,4-Thiadiazole Sulfonamides. Molecules. 15: 9046-9056.

(Received revised manuscript on 12 July 2018) 